\title{
Uniform Expected Utility Criteria for Decision Making under Ignorance or Objective Ambiguity ${ }^{1}$
}

\author{
Nicolas Gravel $^{2}$, Thierry Marchant ${ }^{3}$ and Arunava Sen $^{4}$
}

March 8th 2012

JEL classification numbers: D80, D81.

\footnotetext{
${ }^{1}$ The authors are listed in alphabetic order and deserve equal credit and blame for the content of the paper.

${ }^{2}$ Aix-Marseille University, School of Economics \& GREQAM, Centre de la Vieille Charité, 2, rue de la Charité, 13002 Marseille, France, nicolas.gravel@univ-amu.fr

${ }^{3}$ Ghent University, Department of Data analysis, H. Dunantlaan 1, B-9000 Gent, Belgium, Thierry.Marchant@UGent.be

${ }^{4}$ Indian Statistical Institute, 7, Shaheed Jit Singh Marg, New Delhi, 110016 India, asen@isid.ac.in.
} 


\begin{abstract}
We provide an axiomatic characterization of a family of criteria for ranking completely uncertain and/or ambiguous decisions. A completely uncertain decision is described by the finite set of all its consequences. An ambiguous decisions is described as a finite set of possible probability distributions over a finite set of prizes. Every criterion in the characterized family can be thought of as assigning to every consequence (probability distribution) of a decision an equal probability of occurrence and as comparing decisions on the basis of the expected utility of their consequences (probability distributions) for some utility function.
\end{abstract}




\section{Introduction}

Decision problems are typically categorized by features of the environment assumed to be known to the decision maker. In situations of certainty, the decision maker is assumed to know the unique consequence of every decision. In situations of risk, analyzed for instance in Von Neumann and Morgenstern [1], every decision results in a probability distribution over consequences that is assumed to be known to the decision maker. In situations of uncertainty formalized in Savage [2], a decision or an act is a function from a set of states of nature to a set of consequences. In situations of complete uncertainty or ignorance as studied in the sizeable literature surveyed by [3], a decision is described even more parsimoniously as the set of all its foreseeable consequences, without reference to states of nature or to a process that maps states of nature into consequences. A hybrid category of decision problems is provided by the recent literature on objective ambiguity without state spaces ([4], [5]) where a decision is represented as a set of probability distributions over a set of consequences. This paper is concerned with the two last categories of decisions problems.

It may be useful to illustrate the different types of decision-making problems outlined above. Imagine for this purpose an investor who has to choose between alternative investment strategies that can yield one of three following outcomes: "gaining $\$ 100$ ", "losing $\$ 100 "$ ", and "breaking even (or earning $\$ 0$ )". In the certainty case, each decision leads to a unique consequence (e.g. "gaining $\$ 100$ for sure", "losing $\$ 100$ for sure", and "breaking even for sure"). In the risk model considered by Von Neumann and Morgenstern [1], each decision leads to a probability distribution over these outcomes. For instance decision $A$ could lead to either gaining $\$ 100$ with probability one-half or losing $\$ 100$ with probability one-half while decision $B$ could lead to breaking even with probability one. In the uncertainty model of Savage [2], one could imagine that there are "states of nature" (for instance "High" and "Low" representing whether the stock-market index is rising or falling). In such a setting, decision $A$ is the map that associates $\$ 100$ with the state "High" and $-\$ 100$ with the state "Low". Decision $B$ is the constant map that associates $\$ 0$ with both states. In situations under ignorance, decision $A$ would be represented as the set $\{-100,100\}$ and decision $B$ as the set $\{0\}$. The idea is that the investor recognizes that if she chooses $A$, the outcome will be either $\$ 100$ or $-\$ 100$, but she has no objective basis for assigning likelihoods to the occurrence of either outcome, or for making the outcome depend upon the realization of a "state of nature". ${ }^{1}$ In such a model, the decision maker has a ranking over all decisions, i.e. (finite) subsets of the sets of consequences. Our objective is to provide axioms on the ranking of all such decisions that characterize a class of rules that, we believe, is particularly salient.

As mentioned earlier, there is a large literature on the ranking of sets of objects. The preponderance of rankings considered in this literature are based on the best and the worst consequences of the decisions or on associated lexicographic extensions. ${ }^{2}$ There are two obvious limitations of such "extremist" rankings for understanding decision-making under ignorance. The first is that it is natural to believe (in line with various "expected utility" hypotheses) that decision makers are concerned with "averages" rather than "extremes". For instance, suppose $A=\{\$ 1, \$ 1000000\}$ while $B=\{\$ 0, \$ 900000, \$ 900001, \ldots, \$ 999999\}$. Since the extreme values of $A$ are strictly greater than those of $B$, "extremist" criteria will

\footnotetext{
${ }^{1}$ A very good comparison of the "ignorance" and "uncertainty" setting is done in Pattanaik and Peleg [6].

${ }^{2}$ Notable exceptions are [7] and [8].
} 
favor the former over the latter. However, a convincing case can be made for $B$ over $A$ on the grounds that, on "average" a larger gain is likelier in the former. This is particularly plausible because the decision maker is completely ignorant, i.e. has no model in her mind regarding states of nature and mappings that associate consequences to states. A second drawback of "extremist" rankings is that they do not allow for a diversity of attitudes toward ignorance across decision makers. In situations where decisions have only monetary consequences and all decision makers prefer more money to less, they will all have identical rankings over decisions under positional rules such as maximin, maximax, leximin and so on. This is unsatisfactory since the fact that decision makers have the same preference over certain outcomes should not imply that they have the same attitude toward ignorance. ${ }^{3}$

In this paper, we provide an axiomatic characterization of a family of criteria or decision rules that address these criticisms. The criteria that we characterize are close in spirit to the Expected Utility (EU) family ([1], [2]). They can be viewed as ranking decisions (sets) on the basis of the expected utility of their consequences for some utility function under the assumption that the decision maker assigns an equal probability of occurrence to every consequence of a decision. For this reason we refer to a criterion in this family as a Uniform Expected Utility (UEU) criterion. In the context of the example described earlier, there exist utility numbers for consequences $\$ 100, \$ 0$ and $-\$ 100$ denoted by $u(100), u(0)$ and $u(-100)$ respectively such that $A$ is preferred to $B$ only if $\frac{u(-100)+u(100)}{2}$ is greater than $u(0)$. These rules are clearly based on "averages" rather than "extremes". Moreover, the function $u$ expresses a decision maker's attitude towards ignorance.

Besides the framework of analysis, the main difference between UEU criteria and standard EU ones in [2] lies in the uniformity of the probabilities that our axiomatic structure implies. In our view, uniform probabilities are appropriate in the context of choice under ignorance. A decision maker who ignores the mechanism by which consequences are produced from states of nature and one who is capable only of identifying the set of possible consequences of a decision, has a priori no reason to believe that one consequence is more likely than another. This principle of insufficient reason, renamed the "principle of indifference" by Keynes [9], was the main justification given by early probability theorists such as Bernoulli and Laplace, for their assumption of uniform probabilities in "games of chance" (see also [10] for a recent justification of this principle). Our paper can therefore also be seen as offering an axiomatic characterization of this principle.

The main axioms used in our characterization are Averaging (first introduced in [11]) and Restricted Independence. We provide the characterization in two alternative environments. We first consider a general environment in which no topological property is assumed on the set of possible consequences of a decision. We only assume that the environment is minimally rich (in a sense defined and described in detail in section 2.2). In this setting, we characterize the family of UEU criteria by Averaging, Restricted independence and an Archimedean condition (in the terminology of Krantz, Luce, Suppes and Tversky (KLST) [12]). This general result is then easily applied to a topological setting. We do that in Theorem 3 that characterizes the UEU family in an environment where the set of consequences is a connected separable topological space. Assuming such an environment enables one to eliminate the Richness condition and to replace the Archimedean property by a mild continuity condition on the ranking of decisions.

\footnotetext{
${ }^{3}$ The median-based rankings characterized in [8] are also subject to this difficulty although they avoid the criticism of been based on "extreme" values.
} 
We mentioned above that our results apply also to the literature on objective ambiguity. In this literature, decision makers rank sets of lotteries over consequences (rather than sets of consequences). These sets of lotteries are interpreted as describing "ambiguous" decisions like those arising in the well-known Ellsberg paradox where the decision maker does not know the particular probability distribution over a set of outcomes that is associated with a given decision. In this setting, [4] characterizes a family of criteria which, interpreted in the finite setting considered here, contains the UEU family as a particular case. ${ }^{4}$ The analysis in [4] bears many formal similarities with the somewhat non-standard Bolker-Jeffrey approach to decision making under uncertainty (see e.g. [13], [14], [15]; [16] contains a good discussion of this approach). A common feature of [4], [13], [14] and [15] is that they consider sets (other than singletons considered in [4] but excluded in the others) that contain a continuum of elements. By contrast we limit attention to finite sets of lotteries. This makes the two settings quite different and, among other things, prevents one from viewing our main result as a particular case of theirs. We believe that our finite subsets framework is an important one conceptually. It is, for instance, more suitable for addressing the Ellsberg paradox where the ambiguity concerning the number of balls in an urn is clearly of finite nature.

In addition, there are other environments where our model and results have a natural interpretation. Consider for instance a situation where a jury member has to select one of several possible candidates for a prize or an award (for instance, a film for an Oscar). The prize can be shared by several candidates. One can imagine the jury member ranking all her possible options, in this case being the set of all non-empty subsets of candidates. We believe that our axioms are very plausible in this setting in which case our results will imply that the jury's member's ranking will be a member of the UEU family. In other words, she would have a "utility number" associated with each candidate. In order to compare candidate sets $A$ and $B$, she will compare the (symmetric) average utility of the candidates in the two sets. This would appear to be a reasonable procedure. We note that several papers $([17],[18],[19])$ in mechanism design theory have investigated the strategic behavior of agents who have preferences based on UEU criteria. In the situation described above, imagine that there are several jury members who have to jointly make the selection of the set of winners. Each jury member has an ordering which is a member of the UEU family. However the utility function of each jury member is private information and has to be revealed by each member. A social choice correspondence maps tuples of utility function announcements to sets of winners. The goal of these papers is to investigate the incentive properties of social choice correspondences. The UEU family has also been considered by [20] in their analysis of Nash consistent representation of effectivity functions.

The plan of the rest of this paper is as follows. In the next section, we present the formal framework, the axioms and the family of rankings. The third section presents the main results in the general algebraic setting while the fourth section derives them in a topological environment. The fifth section discusses some implications of the usual notions of comparative uncertainty or ambiguity aversion for UEU criteria. The sixth section comments on the independence of the axioms and the last section concludes.

\footnotetext{
${ }^{4} \mathrm{~A}$ related paper on objective ambiguity is [5].
} 


\section{Notation and basic concepts}

\subsection{Notation}

The sets of integers, non-negative integers, real numbers and non-negative real numbers are denoted respectively by $\mathbb{N}, \mathbb{N}_{+}, \mathbb{R}$ and $\mathbb{R}_{+}$. The cardinality of any set $A$ is denoted by $\# A$ and the $k$-fold Cartesian product of a set $A$ with itself is denoted by $A^{k}$. By a binary relation $\succsim$ on a set $\Omega$, we mean a subset of $\Omega \times \Omega$. Following the convention in economics, we write $x \succsim y$ instead of $(x, y) \in \succsim$. Given a binary relation $\succsim$, we define its symmetric factor $\sim$ by $x \sim y \Longleftrightarrow x \succsim y$ and $y \succsim x$ and its asymmetric factor $\succ$ by $x \succ$ $y \Longleftrightarrow x \succsim y$ and not $(y \succsim x)$. A binary relation $\succsim$ on $\Omega$ is reflexive if the statement $x$ $\succsim x$ holds for every $x$ in $\Omega$, is transitive if $x \succsim z$ always follows $x \succsim y$ and $y \succsim z$ for any $x, y, z \in \Omega$ and is complete if $x \succsim y$ or $y \succsim x$ holds for every distinct $x$ and $y$ in $\Omega$. A reflexive, transitive and complete binary relation is called an ordering.

\subsection{Basic concepts}

Let $X$ be the set of objects. To motivate the framework in terms of decision making under ignorance, we will refer to these objects as possible "consequences" of a decision. In the "objective ambiguity" interpretation of the model, these consequences will be lotteries defined on a more fundamental space of consequences or "prizes". While we do not make any specific assumptions on $X$, it will be clear subsequently that our axioms makes it natural to regard this set as infinite. As an example, one could think of $X$ as being $\mathbb{R}$, interpreted as the set of all conceivable financial returns (either negative or positive) of some investment decision in a highly uncertain environment. As another (objective ambiguity) example, one could think of $X$ as the set of all conceivable probability distributions (lotteries) on a basic set of $k$ different prizes.

We denote by $\mathcal{P}(X)$ the set of all non-empty finite subsets of $X$ (with generic elements $A, B, C, D$, etc.). Any such subset is interpreted as a description of all consequences of a completely uncertain decision or, simply, as a decision. A certain decision with consequence $x \in X$ is identified by the singleton $\{x\}$.

Let $\succsim$ (with asymmetric and symmetric factors $\succ$ and $\sim$ respectively) be an ordering on $\mathcal{P}(X)$. We interpret the statement $A \succsim B$ as meaning "decision with consequences in $A$ is weakly preferred to decision with consequences in $B$ ". A similar interpretation is given to the statements $A \succ B$ ("strictly preferred to") and $A \sim B$ ("equivalent to"). For any ordering $\succsim$ on $\mathcal{P}(X)$, we denote by $\succsim_{S}$ the ordering of $X$ induced by the restriction of $\succsim$ to singletons (that is $x \succsim_{S} x^{\prime} \Longleftrightarrow\{x\} \succsim\left\{x^{\prime}\right\}$ ).

We want to identify the properties (axioms) of the ordering $\succsim$ that are necessary and sufficient for the existence of a function $u: X \rightarrow \mathbb{R}$ such that, for every $A$ and $B$ in $\mathcal{P}(X)$ :

$$
A \succsim B \Longleftrightarrow \sum_{a \in A} \frac{u(a)}{\# A} \geq \sum_{b \in B} \frac{u(b)}{\# B}
$$

An ordering satisfying this property could therefore be thought of as resulting from the comparisons of the expected utility of the consequences of the decision for some utility function under the assumption that the decision maker assigns to every consequence of a decision an equal probability of occurrence. We note that different utility functions $u$ may induce different rankings. We refer to any ranking that satisfies (1) for some function $u$ as to a Uniform Expected Utility (UEU) criterion. 
We now introduce the three axioms that characterize the family of UEU criteria. The first of these axioms, called Averaging, is stated as follows.

Axiom 1 (Averaging) For all disjoint sets $A$ and $B \in \mathcal{P}(X), A \succsim B \Leftrightarrow A \succsim A \cup B \Leftrightarrow$ $A \cup B \succsim B$.

This axiom asserts that enlarging a set $A$ with a (disjoint) set $B$ is worth doing (resp. not worth doing) if and only if the set $B$ of added consequence is better (resp. worse) than the set $A$ to which it is added. It captures an intuitive property satisfied by calculations of "average" in various settings (e.g. adding a student to a class will increase the average of the class if and only if the grade of the added student is larger than the average of the class). The "only if" part of the axiom is strong since it asserts that the only reason for ranking a set $B$ above (resp. below) a set $A$ is when the addition of $B$ to $A$ is considered a good (resp. bad) thing. The Averaging axiom is a compact version of the four Averaging conditions AC1-AC4 discussed in [11] and shown by him to be implied by the UEU family of criteria (as well as by a variant of the additivity axiom of [21], [22] and [23]). The Averaging axiom has been used also in [7] for a characterization of the average Borda ranking of sets as well as by [4] and [13] (see also [14]) in their characterizations for ranking atomless subsets of a universe. A weaker version of Averaging (that only requires the "if" part in its statement) is used in [5]. It is also used in [24] for ranking menus of alternatives in a way that reflects temptation and self-control.

It is also worth mentioning that the Averaging axiom implies the Gärdenfors [25] principle discussed at length in the literature on ignorance as surveyed in [3]. The Gärdenfors principle can be stated formally as follows.

Condition 1 (Gärdenfors Principle) For all $A \in \mathcal{P}(X),(x \in X \backslash A$ and $\{x\} \succ\{a\}$ for all $a \in A) \Rightarrow A \cup\{x\} \succ A$ and $(y \in X \backslash A$ and $\{a\} \succ\{y\}$ for all $a \in A) \Rightarrow A \succ A \cup\{y\}$.

This principle says that it is always (resp. never) worth adding to a set a consequence which, if certain, is better (resp. worse) than all consequences in the set. For future reference we record (without proof) in the following proposition, the fact that the Averaging axiom implies the Gärdenfors principle when applied to a transitive ranking of $\mathcal{P}(X)$.

Proposition 1 Let $\succsim$ be a transitive binary relation on $\mathcal{P}(X)$ that satisfies Averaging. Then $\succsim$ satisfies the Gärdenfors principle.

The second axiom that enters into the characterization of the family of UEU rankings is the following Restricted Independence axiom.

Axiom 2 (Restricted Independence) For all $A, B$ and $C \in \mathcal{P}(X)$ satisfying $\# A=\# B$ and $A \cap C=B \cap C=\emptyset, A \succsim B \Leftrightarrow A \cup C \succsim B \cup C$.

This axiom requires that the ranking of sets with the same number of elements be independent of any elements that they have in common. Adding or subtracting these common elements from the two sets should not affect their ranking. A weak form of the Restricted Independence condition, applied only to the case where $A$ and $B$ are singletons, plays an important role in [26] and [27]. It is worth noticing that the scope of this independence axiom is significantly restricted by the fact that it applies only to sets that have the same number of elements. 
The third and final axiom in our characterization is the following one that we call "Archimedean" since it is similar in spirit to axioms of that name discussed in KLST $[12]$.

Axiom 3 (Archimedean) Let $a, b \in X$ be such that $\{a\} \succ\{b\}$. Consider a sequence of consequences $\left\{c_{i}\right\}, i=1,2, \ldots$ such that either $\left\{c_{i}, a\right\} \sim\left\{c_{i+1}, b\right\}$ for all $i, i+1$ with $i=1,2, \ldots$ or $\left\{c_{i+1}, a\right\} \sim\left\{c_{i}, b\right\}$ for all $i, i+1$ with $i=1,2, \ldots$ holds. Assume further that all elements of the sequence are distinct from $a$ and $b$. If the sequence is strictly bounded by $x$ and $y \in X$ in the sense that $\{x\} \succ\left\{c_{i}\right\} \succ\{y\}$ for every $i$, then the sequence must be finite.

While appearing involved, this axiom is quite simple. Consider two consequences $a$ and $b$ where $a$ is strictly better than $b$. Imagine now that the decision maker is indifferent between a decision leading to either $a$ or some consequence $c_{1}$ and a decision leading to either $b$ or to some consequence $c_{2}$. Intuitively, for such an indifference to arise, $c_{2}$ (added to the bad consequence $b$ ) must be better than $c_{1}$ (added to the good consequence $a$ ). Imagine also that the decision maker is indifferent between decisions $\left\{a, c_{2}\right\}$ and $\left\{b, c_{3}\right\}$ for some other consequence $c_{3}$. Again such an indifference suggests that $c_{3}$ is better than $c_{2}$. When combined with the previous indifference (e.g. between $\left\{a, c_{1}\right\}$ and $\left\{b, c_{2}\right\}$ ), this indifference also suggests that $c_{1}$ and $c_{2}$ on the one hand and $c_{2}$ and $c_{3}$ on the other are "equally spaced" in the preference ordering of the decision maker. Indeed both $c_{1}$ and $c_{2}$ and $c_{2}$ and $c_{3}$ "calibrate" the decision maker's preference for $a$ over $b$. Suppose now that one can construct a sequence of arbitrary length (finite or not) of consequences like that (e.g. $c_{1}, c_{2}, c_{3}, \ldots$ ) that are increasing (or decreasing) and "equally spaced" in this sense. The axiom requires that if the sequence formed by these consequences is infinite, then it can not be bounded. Such a property would be satisfied if the objects were real numbers since any infinite and monotonic sequence of equidistant real numbers is unbounded. This axiom can be considered to be mild since it "bites" only when there exist sequences of the type described above (such sequences are called "standard sequences" in the measurement theory literature). The axiom is trivially satisfied if $X$ is a finite set. In the next section, we will show that this axiom can be replaced by a continuity condition if a topological structure is imposed on $X$.

We now formally state that these three axioms are satisfied by any UEU criterion.

Proposition 2 Any UEU criterion satisfies Averaging, Restricted Independence and the Archimedean axiom.

Proof. It is straightforward to verify that a UEU criterion satisfies Averaging and Restricted Independence. To prove that it satisfies the Archimedean axiom, let $\succsim$ on $P(X)$ be a UEU criterion and consider a sequence of consequences $\left\{c_{i}\right\}$ for $i=1,2, \ldots$ such that, for some consequences $a$ and $b$ distinct from every element in the sequence satisfying $\{a\} \succ\{b\}$, one has, say, $\left\{c_{i}, a\right\} \sim\left\{c_{i+1}, b\right\}$ for all $i=1,2, \ldots$ (the argument is similar if $\left\{c_{i+1}, a\right\} \sim\left\{c_{i}, b\right\}$ for all $\left.i=1,2, \ldots\right)$. Since $\succsim$ is a UEU criterion, there exists a function $u: X \rightarrow R$ such that $u(a)>u(b)$ and $u\left(c_{i}\right)+u(a)=u\left(c_{i+1}\right)+u(b) \Leftrightarrow u(a)-u(b)=$ $u\left(c_{i+1}\right)-u\left(c_{i}\right)$ for all $i$. Assume that the sequence is strictly bounded by $x$ and $y \in X$ so that $\{x\} \succ\left\{c_{i}\right\} \succ\{y\}$ for all $i$. Since $\succsim$ is a UEU criterion, we have $u(x)>u\left(c_{i}\right)>u(y)$. Define the numbers $u_{i}$ by $u_{i}=u\left(c_{i}\right)$ and $d$ by $d=u(a)-u(b)>0$. We therefore have $a$ sequence of numbers $\left.u_{i}, i=1, ..\right)$ such that $u_{i}=(i-1) d+u_{1}>u(y)$ for every $i$ for some strictly positive real number $d$. Clearly one can only have $u(x)>(i-1) d+u_{1}$ for all $i$ of the increasing sequence $\left\{(i-1) d+u_{1}\right\}$ only if the sequence is finite. 
We will show that Averaging, Restricted Independence and the Archimedean axiom characterize the family of UEU rankings of sets if some structure is imposed on the environment. We provide this structure by imposing another axiom on the pair $\langle X, \succsim\rangle$. This axiom imposes richness and smoothness on both the set $X$ of alternatives and the ordering $\succsim$. However this axiom is not specifically tailored to UEU criteria and may be violated by these criteria if the set $X$ of alternatives is "sparse". Theorem 3 below establishes that the axiom can be dispensed with if $X$ is taken to be a connected separable topological space. The formal statement of the Richness axiom is as follows.

Axiom 4 (Richness) For every set $B \in \mathcal{P}(X) \subset X$ and every finite (possibly empty) subset $A$ of $X$, if there exist $c^{*}, c_{*} \in X$ such that $A \cup\left\{c^{*}\right\} \succsim B \succsim A \cup\left\{c_{*}\right\}$, then there exists $c \in X$ such that $A \cup\{c\} \sim B$.

This axiom states that the universe is sufficiently rich to enable, by adding single consequences to sets, various kinds of comparisons with the ordering $\succsim$. Suppose that, starting with decision $B$ and some (possibly empty) set $A$, it is possible to add consequences $c^{*}$ and $c_{*}$ to $A$ in such a way that $A$ enlarged with $c^{*}$ is ranked above $B$ and $A$ enlarged with $c_{*}$ is ranked below $B$. Then it must also be possible to add a consequence $c$ to $A$ such that the resulting set of consequences is indifferent to $A$. The axiom is weak in the sense that the asserted existence of the consequence $c$ is contingent upon the existence of consequences $c^{*}$ and $c_{*}$ that have the required properties.

We emphasize that Richness applies also if the set $A$ to which the consequences $c^{*}$, $c_{*}$ and $c$ are added is empty. Because of this, the axiom implies, in conjunction with the Gardenförs principle, that every decision has a "certainty equivalent". Put differently, an ordering that satisfies Averaging and Richness has the property that every decision has an equivalent "certain decision" (a singleton). For further reference, we formally state this "Certainty Equivalence" condition and the fact that it is implied by Richness if the ranking satisfies Averaging as follows.

Condition 2 (Certainty Equivalence) For every $B \in \mathcal{P}(X)$, there exists $b \in X$ such that $\{b\} \sim B$.

Proposition 3 Let $\succsim$ be an ordering on $\mathcal{P}(X)$ satisfying Averaging and Richness. Then $\succsim$ satisfies the Certainty Equivalence condition.

Proof. Let $B$ be any set in $P(X)$. Because $B$ is non-empty and finite and $\succsim$ is an ordering on $P(X)$, there exists $c^{*}, c_{*} \in B$ (not necessarily distinct) such that $\left\{c^{*}\right\} \succsim\{b\}$ for all $b \in B$ and $\{b\} \succsim\left\{c_{*}\right\}$ for all $b \in B$. By Averaging we have $\left\{c^{*}\right\} \succsim B \succsim\left\{c_{*}\right\}$ which can be written equivalently as $\varnothing \cup\left\{c^{*}\right\} \succsim B \succsim \varnothing \cup\left\{c_{*}\right\}$. By Richness (for $A=\varnothing$ ), there exists c such that $\{c\} \cup \varnothing \sim B$, which proves Certainty Equivalence.

It is also important to note that the combination of the Richness and Averaging axioms implies that either the ranking $\succsim$ is trivial or that there are infinitely many consequences in $X$. Specifically, if $X$ is finite, then a decision maker who compares decisions in $\mathcal{P}(X)$ with an ordering satisfying Averaging and Richness (and therefore Certainty Equivalence, due to Proposition 3) must be indifferent between all decisions. We state this formally as follows.

Proposition 4 Suppose $\# X=n$ for some $n \in \mathbb{N}_{+}$and let $\succsim$ be an ordering on $\mathcal{P}(X)$ satisfying Averaging. Then $\succsim$ satisfies Certainty Equivalence if and only if $A \sim B$ for all 
Proof. It is clear that the trivial ordering defined by $A \sim B$ for all $A, B \in P(X)$ satisfies Certainty Equivalence (as well as Averaging). To prove the reverse implication, write the finite set $X$ as $X=\left\{x_{1}, \ldots, x_{n}\right\}$ and assume without loss of generality (since $\succsim$ is an ordering) that $\left\{x_{i}\right\} \succsim\left\{x_{i+1}\right\}$ for $i=1, \ldots, n-1$. By Averaging, we must have, for every $i=1, \ldots, n-1$ :

$$
\left\{x_{i}\right\} \succsim\left\{x_{i}, x_{i+1}\right\} \succsim\left\{x_{i+1}\right\}
$$

By certainty equivalence there exists $x(i) \in X$ such that $x(i) \sim\left\{x_{i}, x_{i+1}\right\}$. Either $\{x(i)\} \succsim$ $\left\{x_{i}\right\}$ or $\left\{x_{i+1}\right\} \succsim\{x(i)\}$. In the first case, one has $\left\{x_{i}, x_{i+1}\right\} \sim\{x(i)\} \succsim\left\{x_{i}\right\}$ so that, by Averaging and transitivity, $\left\{x_{i}\right\} \sim\left\{x_{i+1}\right\}$. In the other case, one has $\left\{x_{i+1}\right\} \succsim\{x(i)\} \sim$ $\left\{x_{i}, x_{i+1}\right\}$ so that, again, the conclusion $\left\{x_{i}\right\} \sim\left\{x_{i+1}\right\}$ follows from Averaging and transitivity. Hence all pairs and singletons must be indifferent. Repeated application of Averaging (adding first indifferent singletons to pairs and then indifferent singletons to triples etc.) leads to the conclusion of universal indifference.

We note in passing that the trivial ordering that considers all sets to be indifferent is a member of the UEU family (any constant function $u$ having $X$ as domain could serve as a representation as per (1)). Hence, in the rest of the paper, we shall be interested in characterizing the UEU family of orderings of $\mathcal{P}(X)$ in the non trivial case where there are at least two sets $A$ and $B$ such $A \succ B$.

Beside forcing $X$ to be infinite or $\succsim$ to be trivial (when combined with Averaging), the Richness axiom precludes from consideration some "discontinuous" rankings such as the "Leximin" and the "Leximax" rules studied in [6]. For instance, the Leximin rule compares sets on the basis of their worst consequences. If a tie in the worst consequence arises, then the second worst consequence is considered and so on until either a strict ranking is obtained or the consequences in at least one of the sets are exhausted. In the latter case the set which contains the largest number of elements is ranked higher. Such a rule violates Richness. Indeed if we take $X=\mathbb{R}_{+}$one has that $\{1,3\} \prec\{2\} \prec\{2,3\}$ for the Leximin criterion. Yet, contrary to what is required by Richness, it is impossible to find a non-negative real number $x$ such that $\{x\} \cup\{3\} \sim\{2\}$.

It should be also noted that the Richness axiom is not specifically related to the UEU family and may even be violated by a UEU criterion if the set $X$ is not sufficiently rich. Suppose $X=\mathbb{N}$ and the function $u$ of $(1)$ is the identity function. Since $\frac{2+6+7}{3}=5<$ $\frac{5+6}{2}=5.5<\frac{2+6+10}{3}=6$, we have $\{2,6,7\} \prec\{5,6\} \prec\{2,6,10\}$. Yet, contrary to what Richness requires, there does not exist an integer $c$ such that $\frac{2+6+c}{3}=\frac{5+6}{2}$. Hence there does not exist a consequence $c$ such that $\{2,6, c\} \sim\{5,6\}$.

\section{Main results}

The main result proved in this paper is the following.

Theorem 1 Let $\succsim$ be a non-trivial ordering on $\mathcal{P}(X)$ satisfying Richness. Then $\succsim$ satisfies Averaging, Restricted Independence and the Archimedean axiom if and only if it is a UEU criterion. Furthermore, the $u$ function in the definition of a UEU criterion is unique up to a positive affine transformation. 
The general logic underlying the proof of this result, detailed in the Appendix, is as follows.

First, we establish the result on a subdomain of $\mathcal{P}(X)$ consisting of all finite subsets of $X$ that exclude minimal and maximal (with respect to $\succsim_{S}$ ) elements of the universe (if any). Let us denote by $X^{\prime}$ this set and by $\mathcal{P}\left(X^{\prime}\right)$ the set of all finite subsets of $X^{\prime}$ (the formal definitions of these is done in the Appendix). After having obtained that any ordering on $\mathcal{P}\left(X^{\prime}\right)$ satisfying Averaging, Restricted Independence, the Archimedean axiom and Richness can be represented as per (1) for some function $u: X^{\prime} \rightarrow \mathbb{R}$, we then show that this numerical representation can be "extended" to the whole set $\mathcal{P}(X)$.

The proof of the restricted version of Theorem 1 on $\mathcal{P}\left(X^{\prime}\right)$ is made of two main steps. In the first, we show that Averaging, Restricted Independence and the Archimedean axiom characterize the family of UEU criteria in an environment satisfying Richness if one restricts attention to subsets of $X^{\prime}$ that have at most two consequences. The proof of this result, also provided in the Appendix, critically rests on Theorem 2 of KLST [12] (p. 257 ) on additively separable numerical representations of an ordering over a Cartesian product $X \times X$ (see also [28] or [29] for an earlier statement framed in a topological setting). An important ingredient in the proof is the demonstration that the ranking of the Cartesian product $X \times X$ induced by $\succsim$ in our axiomatic structure, satisfies the so-called "Thomsen condition" (see KLST [12], Definition 3, p. 250). This is shown in Lemma 2 of the Appendix.

The characterization result for decisions involving at most two distinct consequences that is proved in the Appendix is the following.

Theorem 2 Let $\succsim$ be a non-trivial ordering on $\mathcal{P}(X)$ satisfying Richness. Then, if $\succsim$ satisfies Averaging, Restricted Independence and the Archimedean axiom, its restriction to the sets in $\mathcal{P}\left(X^{\prime}\right)$ of cardinality no larger than 2 can be represented as per (1) for some utility function $u: X^{\prime} \longrightarrow \mathbb{R}$. Furthermore, the utility function $u$ is unique up to a positive affine transformation.

The second step extends Theorem 2 to subsets of $X^{\prime}$ with an arbitrary (but finite) number of consequences using the same axioms. Specifically, we prove in the Appendix that the unique utility function whose expectation (under uniform probabilities) represents the ranking of sets containing at most two elements exhibited in Theorem 2 also represents the ranking of sets of larger cardinality. A key step in the argument is the technical Lemma 4, stated and proved in Appendix, that enables one to approximate the arithmetic mean of a set of $n$ numbers recursively from the arithmetic means of pairs of those numbers.

Using this lemma, we show in the Appendix that the evaluation of any decision involving more than two consequences can be made on the basis of formula (1) if the evaluation of all decisions involving at most two consequences can be made on that basis. The argument works iteratively by "squeezing" the certainty equivalent of any decision $A$ having more than two consequences between successive pairs (one above and one below) of decisions, each with two consequences. These two consequences are either an element of $A$ or the certainty equivalent of a previous decision in the sequence. The successive pairs of decisions with two consequences can be evaluated by (1) thanks to Theorem 2 and they are constructed in such a way that their average utilities behave just like the sequences of arithmetic means of two numbers studied in Lemma 4. Thanks to this lemma, we can then know that the utility of the certainty equivalent of $A$ is equal to 
the average utility of the elements in $A$. We can therefore use this average utility to numerically represent the ordering $\succsim$ on all finite subsets of $X^{\prime}$, giving us the required restricted version of Theorem 1 (called Theorem 4 in the Appendix).

The final step of the proof consists in showing that the numerical representation of $\succsim$ restricted to $\mathcal{P}\left(X^{\prime}\right)$ of Theorem 4 can be extended to the whole set $\mathcal{P}(X)$ so as to give Theorem 1.

\subsection{Related literature}

There are two papers that are immediately related to ours. The first is [11] which characterizes the UEU family of rankings of all non-empty subsets of a finite universe. The model does not impose any restriction on the environment. As a result, the characterization is a direct adaptation of the additivity axiom of [21], [22] and [23]. The unappealing nature of this axiom is well-known and is especially striking when adapted to the problem of comparing sets on the basis of their average utility. The additivity axiom involves the construction of arbitrarily long sequences of set comparisons which are both difficult to motivate as primitive axioms as well as hard to verify in practice. By contrast, the structure of our model enables us to characterize the UEU family of rankings of sets by means of axioms that are, in our opinion, easier to interpret and verify. We note that our Averaging axiom is identified in [11] as being satisfied by any ranking in the UEU family. Our contribution is to show that in conjunction with Restricted Independence and the Archimedean axiom, Averaging characterizes the UEU family in a sufficiently rich environment.

The other related paper is [7]. It provides a characterization of a ranking of finite sets based on the average Borda score of their elements. This ranking is clearly a member of the UEU family for which the utility of a consequence is defined by its Borda score. Our paper, in contrast provides a characterization of the entire family of UEU criteria. Like [11], [7] uses the Averaging axiom (along with several others) but do not use an Archimedean axiom or any richness condition.

\section{Topological interpretation of the structural envi- ronment}

We show in this section that if we impose a topological structure on the set $X$ from the outset, then the Richness axiom can be dispensed with entirely. Moreover, the Archimedean axiom can be replaced by a mild continuity one in the characterization. This shows that the algebraic structure considered in the previous section is more general for the purpose at hand than the standard topological structure assumed in many decision problems (see e.g. [30] for similar findings in a different context).

Assume specifically that $X$ is a connected subset of a separable topological space. ${ }^{5}$ At least two interpretations could be given to $X$ in this context. First, $X$ could be thought of as the set of all bundles of $k$ goods that could result from any uncertain decision. Here it would be natural to take $X=\mathbb{R}^{k}$ interpreting it as all bundles of goods. The case of

\footnotetext{
${ }^{5} \mathrm{~A}$ subset $A$ of a topological space is connected if it can not be written as a finite union of pairwise disjoint open sets. A topological space $S$ is separable if it contains a countable subset the closure of which is $S$.
} 
$k=1$ could be interpreted as the special case of decisions with monetary consequences that we have discussed earlier.

The second interpretation, in line with the literature on objective ambiguity (see e.g. [4] or [5]) is to view $X$ as the set of all lotteries yielding $k$ different prizes. A typical element $p \in X$ is a probability vector assigning to every prize $h$ (for $h=1, \ldots, k$ ) its probability of realization $p_{h} \in[0,1]$. A finite set $A \subset X$ of such probability vectors is then an ambiguous decision in which the precise probability distribution over the set of $k$ prizes is not known to the decision maker. A classical instance of decision making under this kind of ambiguity is provided by the so-called Ellsberg paradox in which a decision maker does not know how a certain number of balls are split between two colors (see [5] for further discussion). If this interpretation is favored, then $X=S^{k-1}=\left\{p \in[0,1]^{k}: \sum_{j=1}^{k} p_{j}=1\right\}$. 6

For either of these interpretations, we believe that it is natural to impose the following Continuity axiom on $\succsim$.

Axiom 5 (Continuity) For all $A \in \mathcal{P}(X)$, the sets $B(A)=\{x \in X:\{x\} \succsim A\}$ and $W(A)=\{x \in X: A \succeq\{x\}\}$ are closed in $X$.

According to the axiom, a small change in a consequence (lottery) should not have a drastic effect on the ranking of this consequence (lottery) vis-à-vis any set. We emphasize that our Continuity axiom only concerns comparisons of sets vis-à-vis singletons. It is therefore much weaker than the (Vietoris) continuity condition used for instance in [26] which restricts the comparisons of two sets in a way that is not even compatible with the UEU family of set rankings. It is clear that any UEU criterion for which the associated $u$ function is continuous, satisfies this axiom.

The result in this section establishes that the UEU family is characterized by Averaging, Restricted Independence and Continuity in this environment. In view of Theorem 1 , we only need to prove that, if $X$ is a connected subset of separable topological space, then an ordering that satisfies Continuity, Averaging and Restricted Independence also satisfies the Richness and the Archimedean axioms.

Theorem 3 Let $X$ be a connected subset of a separable topological space and let $\succsim$ be an ordering of $\mathcal{P}(X)$. Then $\succsim$ satisfies Continuity, Averaging and Restricted Independence if and only if it is a UEU criterion for which the function $u$ is continuous.

As before, the proof of Theorem 3 is provided in the Appendix. Below, we make some remarks about the literature related to the ranking of subsets of some topological spaces.

\subsection{Related Literature}

A paper using a topological framework but arriving at strikingly different results is [26]. This paper characterizes rankings that compare sets on the basis of their maximal and minimal elements using Hausdorff continuity and a mild independence condition. Our UEU criteria satisfy the independence condition but violate Hausdorff continuity. Continuity is not a straightforward notion when applied to a ranking of sets of objects (as opposed to a ranking of objects). We believe that our notion of continuity is very natural and that of Hausdorff continuity rather restrictive. For instance, it seems very persuasive

\footnotetext{
${ }^{6}$ Here $S^{k-1}$ is the unit simplex of dimension $k-1$.
} 
to regard members of the UEU family (defined with respect to a continuous function $u$ ) as being continuous.

As mentioned in the Introduction, UEU type criteria have also been considered in the literature on decision under objective ambiguity. For instance, [4] (see also [13]) characterizes all rankings of the following kind: for all sets $A$ and $B$ in the domain,

$$
A \succsim B \Longleftrightarrow \frac{\int_{A} u(a) d \mu}{\mu(A)} \geq \frac{\int_{B} u(b) d \mu}{\mu(B)}
$$

for some function $u$ and some probability measure $\mu$ defined on an algebra of atomless subsets of $X$. The UEU family could be considered to be a sub-family of this class of orderings if the measure $\mu$ could be taken to be the cardinality of the sets. Yet, [4] and [13] exclude finite sets from their domain, while we do not consider non-finite sets. Hence our results are completely independent from theirs. As we mentioned earlier, we believe that the finite setting in which we obtain our characterization is a clear advantage of our approach, notably with respect to possible empirical or experimental verification. It is, indeed, rather difficult to construct experimental settings in which a decision maker is confronted with decisions with a continuum of consequences.

\section{Comparative ambiguity/uncertainty aversion}

Ambiguity aversion is sometimes perceived as the main reason for the preference pattern observed in the so-called Ellsberg paradox and uncertainty aversion can be seen as lying behind the so-called "precautionary principle" in public decision making. It is therefore of some interest to have an easy diagnostic tool for appraising the degree of uncertainty/ambiguity aversion of a decision maker placed in an ambiguous or radically uncertain environment. We now briefly show that such a diagnostic is particularly easy for UEU criteria.

We start with the following definition of what it means for a binary relation $\succsim^{1}$ to exhibit at least as much uncertainty/ambiguity aversion as a binary relation $\succsim^{2}$ (see also [4] and [5] for similar definitions in the specific context of decision making under objective ambiguity).

Definition 1 Comparative ambiguity/uncertainty aversion. Given two binary relations $\succsim^{1}$ and $\succsim^{2}$ defined on $\mathcal{P}(X)$, we say that $\succsim^{1}$ is at least as ambiguity/uncertainty averse as $\succsim^{2}$ if, for every consequence $x \in X$ and any decision $A \in \mathcal{P}(X),\{x\} \succsim^{2} A \Rightarrow\{x\} \succsim^{1} A$ and $\{x\} \succ^{2} A \Rightarrow\{x\} \succ^{1} A$.

In words, a decision maker with preference $\succsim^{1}$ is at least as uncertainty/ambiguity averse as a decision maker with preference $\succsim^{2}$ if it is the case that whenever the latter prefers (weakly or strictly) a certain (non-ambiguous) decision to some other decision, then so does the former. The following proposition, whose easy proof is provided in the Appendix, establishes the implication of this definition in the case of two decision makers with UEU preferences.

Proposition 5 Let $\succsim^{1}$ and $\succsim^{2}$ be two orderings on $\mathcal{P}(X)$ which can be represented as per (1) for some functions $u^{1}$ and $u^{2}$ respectively (both having $X$ as domain and $\mathbb{R}$ as range). Then $\succsim^{1}$ is at least as ambiguity/uncertainty averse as $\succsim^{2}$ as per definition 1 if and only if there exists some increasing and concave function $\Psi$ having the image of $\mathbb{R}$ under $u^{2}$ as domain and $\mathbb{R}$ as range such that $u^{1}(x)=\Psi\left(u^{2}(x)\right)$. 
According to Proposition 5, the statement "is at least as uncertainty/ambiguity averse as" in the class of UEU orderings is equivalent to the statement "has a weakly more concave utility function than". This is clearly the counterpart of increasing risk aversion in the standard vNM theory of choice under risk (see for instance, Chapter 6 in [31]).

We conclude this section by mentioning an implication of this discussion for the interpretation of our model in terms of decision making under objective ambiguity, when the decision maker can be assumed to have conventional VNM preferences over lotteries. Suppose therefore that $X=S^{k-1}$ and that, in addition to the axioms considered in the previous section, we impose on the binary relation $\succsim$ the following "Singleton Independence" axiom.

Axiom 6 Singleton Independence. For every $p, q$ and $r \in S^{k-1}$ and every number $\lambda \in[0,1],\{p\} \succsim\{q) \Longleftrightarrow\{\lambda p+(1-\lambda) r\} \succsim\{\lambda q+(1-\lambda) r\}$.

The following Proposition which restricts the function $u$ that represents UEU ranking in (1) to be additively separable between probabilities, can then be obtained as a corollary of Theorem 3 and standard textbook versions of the Expected Utility Theorem (see e.g. Proposition 6.B.3 in [31]).

Proposition 6 Let $\succsim$ be an ordering on $\mathcal{P}\left(S^{k-1}\right)$. Then $\succsim$ satisfies Averaging, Restricted Independence, Singleton Independence and Continuity if and only if there exists some increasing function $\Phi: \mathbb{R} \rightarrow \mathbb{R}$ and some vector $v \in \mathbb{R}^{k}$ such that

$$
A \succsim B \Longleftrightarrow \sum_{p \in A} \frac{\Phi\left(\sum_{h} p_{h} v_{h}\right)}{\# A} \geq \sum_{q \in B} \frac{\Phi\left(\sum_{h} q_{h} v_{h}\right)}{\# B}
$$

Hence the interpretation of the UEU family of rankings in the context of choice under objective ambiguity enables one, at least in the context of standard vNM theory, to disconnect the attitude toward risk and the attitude toward ambiguity. The attitude toward risk is captured by the functional relation that exists between the numbers $v_{1}, \ldots v_{k}$ in Proposition 6 and the prizes to which they correspond. As established by Proposition 5 , attitude toward ambiguity is captured by the degree of concavity of the function $\Phi$ that aggregates vNM individual utility numbers $v_{1}, \ldots, v_{k}$ into the $u$ function.

\section{Independence of the axioms}

We show that Averaging, Restricted Independence and the Archimedean axioms are logically independent when they are imposed on an ordering that satisfies Richness. Since any UEU criterion satisfies Averaging, Restricted Independence and the Archimedean axiom, it can therefore be said that these three axioms provide a minimal characterization of the UEU family of orderings in any environment where these orderings satisfy Richness. It is clear that these examples can also be used to show the logical independence of Averaging, Restricted Independence and Continuity when $X$ is assumed to be a connected subset of a separable topological space.

Example 1 Let $X=\mathbb{R}_{+}$. For all $A, B \in P(X)$,

$$
A \succsim B \Longleftrightarrow \sum_{a \in A} a \geq \sum_{b \in B} b .
$$


It can be checked easily that this (Cardinality) ranking satisfies Restricted Independence, the Archimedean axiom, Continuity and Richness but violates Averaging. Indeed, $\{3\} \succsim$ $\{1,2\}$ but $\{3\} \prec\{1,2,3\}$.

Example 2 Let $X=\mathbb{R}_{++}$and define $\succsim$ by:

$$
A \succsim B \Leftrightarrow \frac{\sum_{a \in A} a}{\sum_{a \in A} \frac{1}{a}} \geq \frac{\sum_{b \in A} b}{\sum_{b \in A} \frac{1}{b}}
$$

This ordering satisfies the Averaging axiom. It is a continuous ordering because the set $B(A)=\left\{x \in X: x^{2} \geq \sum_{\sum_{a \in A} \frac{1}{a}}\right\}$ and $W(A)=\left\{x \in X: \sum_{\sum_{a \in A} a} a\right.$ set $A \in P(X)$. Moreover it can be seen that it satisfies the Archimedean axiom using an argument that parallels that of Proposition 2. Yet $\succsim$ violates Restricted Independence because if we take

$$
A=\{1,7\}, B=\{2,3\} \text { and } C=\{4,12\}
$$

we have $A \succsim B$ since, using (4):

$$
\frac{1+7}{1+\frac{1}{7}}=7 \geq \frac{2+3}{\frac{1}{2}+\frac{1}{3}}=6
$$

but, contrary to what is required by Restricted Independence, we have $A \cup C \prec B \cup C$ since:

$$
\frac{1+4+7+12}{1+\frac{1}{4}+\frac{1}{7}+\frac{1}{12}}=\frac{24 \times 84}{84+21+12+7}=\frac{6 \times 84}{31}<\frac{2+3+4+12}{\frac{1}{2}+\frac{1}{3}+\frac{1}{4}+\frac{1}{12}}=6 \times 3
$$

Example 3 Let $X=\mathbb{R} \times \mathbb{R}$. For any $a \in X$ and $i \in\{1,2\}$, let $a_{i}$ denote the $i$-th component of a. Define the ordering $\succsim$ by:

- $A \sim B$ iff $\sum_{a \in A} \frac{a_{1}}{\# A}=\sum_{b \in B} \frac{b_{1}}{\# B}$ and $\sum_{a \in A} \frac{a_{2}}{\# A}=\sum_{b \in B} \frac{b_{2}}{\# B}$;

- $A \succ B$ iff either

(i) $\sum_{a \in A} \frac{a_{1}}{\# A}>\sum_{b \in B} \frac{b_{1}}{\# B}$ or

(ii) (ii) $\sum_{a \in A} \frac{a_{1}}{\# A}=\sum_{b \in B} \frac{b_{1}}{\# B}$ and $\sum_{a \in A} \frac{a_{2}}{\# A}>\sum_{b \in B} \frac{b_{2}}{\# B}$. 
The ordering is a lexicographic combination of two UEU orderings defined on each dimension. We first prove that it violates the Archimedean axiom. The set $\{(2, i): i \in Z\}$ is a standard sequence because $\{(2, i),(1,2)\} \sim\{(2, i+1),(1,1)\}$ for all $i \in Z$. This standard sequence is infinite but is bounded. Indeed, for any $i \in N$, we have $\{(3,1)\} \succ$ $\{(2, i)\} \succ\{(1,1)\}$. The ordering can also be verified to violate continuity.

We now show that $\succsim$ satisfies Averaging. Suppose first that $A \succ B$. Using the definition of $\succsim$, this is either equivalent to:

$$
\begin{aligned}
\frac{1}{\# A} \sum_{a \in A} a_{1} & >\frac{1}{\# B} \sum_{b \in B} b_{1} \\
& \Longleftrightarrow \\
\frac{1}{\# A} \sum_{a \in A} a_{1} & >\frac{1}{\# A+\# B} \sum_{\alpha \in A \cup B} \alpha_{1} \\
& \Longleftrightarrow \\
A & \succ A \cup B
\end{aligned}
$$

or to:

$$
\begin{aligned}
\frac{1}{\# A} \sum_{a \in A} a_{1} & =\frac{1}{\# B} \sum_{b \in B} b_{1} \text { and } \frac{1}{\# A} \sum_{a \in A} a_{2}>\frac{1}{\# B} \sum_{b \in B} b_{2} \\
& \Longleftrightarrow \\
\frac{1}{\# A} \sum_{a \in A} a_{1} & =\frac{1}{\# A+\# B} \sum_{\alpha \in A \cup B} \alpha_{1} \text { and } \frac{1}{\# A} \sum_{a \in A} a_{2}>\frac{1}{\# A+\# B} \sum_{\alpha \in A \cup B} \alpha_{2} \\
& \Longleftrightarrow
\end{aligned}
$$$$
A \succ A \cup B \text {. }
$$

$A$ similar reasoning holds when $A \sim B$. To show that $\succsim$ satisfies Richness, consider arbitrary subsets $A$ and $B$ of $X$ (with $A$ possibly empty) and define $c$ by means of the following two equations:

$$
\frac{c_{1}+\sum_{a \in A} a_{1}}{1+\# A}=\frac{\sum_{b \in B} b_{1}}{\# B} \text { and } \frac{c_{2}+\sum_{a \in A} a_{2}}{1+\# A}=\frac{\sum_{b \in B} b_{2}}{\# B} . .
$$

We then have $A \cup\{c\} \sim B$. We notice that this conclusion holds no matter what is assumed on the ranking of $A$ vis-à-vis $B$. Hence this conclusion can also be obtained for sets $B$ and $A$ that satisfy the requirements of the Richness axiom.

Finally, to show that $\succsim$ satisfies Restricted Independence, consider finite and nonempty subsets $A$ and $B$ of $X$ such that $\# A=\# B$ and some finite and non-empty set $C$ such that $A \cap C=\emptyset=B \cap C$. We have $A \succ B$ if and only if either:

$$
\frac{1}{\# A}\left(\sum_{a \in A} a_{1}\right)>\frac{1}{\# B}\left(\sum_{b \in B} b_{1}\right) \Leftrightarrow \frac{1}{\# A+\# C}\left(\sum_{\alpha \in A \cup C} \alpha_{1}\right)>\frac{1}{\# B+\# C}\left(\sum_{\beta \in B \cup C} \beta_{1}\right)
$$




$$
\begin{aligned}
& \Longleftrightarrow \\
\text { or: } \quad & \\
\frac{1}{\# A}\left(\sum_{a \in A} a_{1}\right)=\frac{1}{\# B}\left(\sum_{b \in B} b_{1}\right) & \text { and } \frac{1}{\# A}\left(\sum_{a \in A} a_{2}\right)>\frac{1}{\# B}\left(\sum_{b \in B} b_{2}\right) \\
& \Longleftrightarrow \\
\frac{1}{\# A+\# C}\left(\sum_{\alpha \in A \cup C} \alpha_{1}\right)=\frac{1}{\# B+\# C}\left(\sum_{\beta \in B \cup C} \beta_{1}\right) & \text { and } \frac{1}{\# A+\# C}\left(\sum_{\alpha \in A \cup C} \alpha_{2}\right)>\frac{1}{\# B+\# C}\left(\sum_{\beta \in B \cup C} \beta_{2}\right) \\
& \Longleftrightarrow \\
A \cup C & \succ B \cup C .
\end{aligned}
$$

\section{Conclusion}

This paper characterizes the family of UEU rankings of decisions under ignorance or objective ambiguity under the assumption that the rankings are defined in a rich environment. The axioms used are easy to interpret and, except perhaps for continuity or the Archimedean axiom, are verifiable from observation of choice behavior. We have also shown that UEU rankings can be used to compare ambiguous decisions or decisions with financial consequences and is characterized in these setting by the same axioms but with the Archimedean axiom replaced by a mild Continuity requirement. The framework can also be used to provide an analysis of comparative attitudes toward radical uncertainty or toward ambiguity.

A limitation of UEU criteria is that they assign to every consequence of a decision the same probability of occurrence. A next step in the research agenda is to identify the properties of a more general EU criterion that does not impose this assumption. A natural candidate would be the finite counterpart of the family of orderings described by (2). Members of this family satisfy Averaging and Continuity (or the Archimedean axiom) but, as illustrated in example 2 in section 6, may violate Restricted Independence. Obtaining a satisfactory characterization of this class of rankings in a finite setting strikes us as a high priority for future research.

\section{Appendix}

\subsection{Proof of Theorem 2}

Define the (possibly empty) sets $m(X)$ and $M(X)$ of minimal and maximal (respectively) elements of $X$ with respect to $\succsim_{S}$ by:

$$
\begin{aligned}
& m(X)=\{x \in X:\{x\} \precsim\{y\} \forall y \in X\} \text { and } \\
& M(X)=\{z \in X:\{z\} \succsim\{y\} \forall y \in X\}
\end{aligned}
$$


Let $X^{\prime}$ be defined by $X^{\prime}=X \backslash(m(X) \cup M(X))$. We first show that, under our assumptions, the set $X^{\prime}$ is "unbounded" in the sense that for any $x \in X^{\prime}$, we can find some consequences $w$ and $z \in X^{\prime}$ such that $\{w\} \prec\{x\} \prec\{z\}$. We state and prove this result formally as follows.

Proposition 7 Let $\succsim$ be a non-trivial ordering on $\mathcal{P}(X)$ satisfying Richness and Averaging. Then, $X^{\prime} \neq \emptyset$ and for all $x \in X^{\prime}$, there exist $w, z \in X^{\prime}$ such that $\{w\} \prec\{x\} \prec\{z\}$.

Proof. Since $\succsim$ is non-trivial, there exist $A, B \in \mathcal{P}(X)$ such that $A \succ B$. Consider any $x \in X^{\prime}$. By Proposition 3, the ordering $\succsim$ satisfies Certainty Equivalence. Therefore, there exist $a, b \in X$ such that $A \sim\{a\}$ and $B \sim\{b\}$. By Averaging, $\{a\} \succ\{a, b\} \succ\{b\}$. By Certainty Equivalence, there exists a $c \in X$ such that $\{c\} \sim\{a, b\}$. Clearly, $c \in X^{\prime}$. We now find $w \in X^{\prime}$ such that $\{w\} \prec\{x\}$. Suppose first that $m(X)=\emptyset$. This means that $x \notin m(X)$ so that there exists some $t \in X$ such that $\{t\} \prec\{x\}$. By Certainty Equivalence, there exists some $w \in X$ such that $\{w\} \sim\{t, x\}$. By Averaging and transitivity, $\{x\} \succ\{w\} \succ\{t\}$. Hence $w \notin m(X) \cup M(x)$ so that $w \in X^{\prime}$. Suppose now that $m(X) \neq \emptyset$ and let $t \in m(X)$. By definition of $m(X)$, we have $\{t\} \prec\{x\}$ so that, by Certainty Equivalence again, there exists some $w$ such that $\{w\} \sim\{t, x\}$. As before, we can conclude from Averaging that $\{t\} \prec\{w\} \prec\{x\}$ so that $w \in X \backslash(m(X) \cup M(X))$, as required. The argument for finding some $z \in X^{\prime}$ such that $\{z\} \succ\{x\}$ is similar.

Before proving Theorems 2 and 4 on the sub-domain $\mathcal{P}\left(X^{\prime}\right)$, we must verify that all our axioms - formulated for the domain $\mathcal{P}(X)$ - are also valid for the sub-domain $\mathcal{P}\left(X^{\prime}\right)$. We do this in the following lemma.

Lemma 1 Let $\succsim$ be an ordering on $\mathcal{P}(X)$ satisfying Averaging, Restricted Independence, Richness and the Archimedean axiom. Then the restriction of $\succsim$ to $\mathcal{P}\left(X^{\prime}\right)$ satisfies the same axioms.

Proof. It is easy to verify that this is indeed the case for Averaging, Restricted Independence and the Archimedean axiom. For Richness, let $A$ and $B$ be two finite subsets of $X^{\prime}$ (with $A$ possibly empty) and assume that there are consequences $c^{*}$ and $c_{*} \in X^{\prime}$ such that $A \cup\left\{c^{*}\right\} \succsim B \succsim A \cup\left\{c_{*}\right\}$. By Richness (applied to $\mathcal{P}(X)$ ), there exists a consequence $c \in X$ such that $A \cup\{c\} \sim B$. We need to show that $c \in X^{\prime}$. By contradiction, assume $c \in X \backslash X^{\prime}=m(X) \cup M(X)$. If $c \in m(X)$, then $\{c\} \prec\{x\}$ for all $x \in X^{\prime}$ so that, in particular, $\{c\} \prec\left\{c_{*}\right\}$ and $\{c\} \prec\{a\}$ for all $a \in A$. Hence $c \notin A$. One has therefore, by Restricted Independence (if $c_{*} \notin A$ ) or by Averaging (if $c_{*} \in A$ ), that $B \succsim A \cup\left\{c_{*}\right\} \succ A \cup\{c\}$, a contradiction (if $\succsim$ is transitive). The argument is symmetric if $c \in M(X)$.

An important ingredient in the proof of Theorem 2 is the following lemma, which states that if an ordering $\succsim$ on $\mathcal{P}\left(X^{\prime}\right)$ satisfies Averaging, Restricted Independence, Richness and the Archimedean axiom, then it satisfies, when restricted to pairs and singletons, the following important condition that is closely related to the so-called "Thomsen condition" in the theory of conjoint measurement (using KLST [12] terminology).

Lemma 2 Let $\succsim$ be an ordering on $\mathcal{P}\left(X^{\prime}\right)$ satisfying Averaging, Restricted Independence, Richness and the Archimedean axiom. Then for every (not necessarily distinct) consequences $a, b, c, d$, e and $f \in X^{\prime},\{a\} \cup\{f\} \sim\{c\} \cup\{e\}$ and $\{c\} \cup\{d\} \sim\{b\} \cup\{f\}$ must imply $\{a\} \cup\{d\} \sim\{b\} \cup\{e\}$. 
Proof. We consider several cases.

1. $\{a\} \sim\{b\}$ and $\{d\} \sim\{e\}$. In this case, we conclude that $\{a\} \cup\{d\} \sim\{b\} \cup\{d\}$ by Restricted Independence if both $a \neq d$ and $b \neq d$. If either $a=d$ and $b \neq d$ or $a \neq d$ and $b=d$, the conclusion $\{a\} \cup\{d\} \sim\{b\} \cup\{d\}$ follows from Averaging. Finally, if $a=d$ and $b=d$, the conclusion that $\{a\} \cup\{d\} \sim\{b\} \cup\{d\}$ follows trivially from the assumption that $\{a\} \sim\{b\}$. By an analogous reasoning we can obtain the conclusion that $\{b\} \cup\{d\} \sim\{b\} \cup\{e\}$. The conclusion that $\{a\} \cup\{d\} \sim\{b\} \cup\{e\}$ follows then at once from transitivity.

2. $\{a\} \succ\{b\}$ and $\{d\} \succsim\{e\}$. In this case, it follows from Restricted Independence (if $a \neq$ $f \neq b$ ) or Averaging (if $a=f$ or $b=f$ ) that $\{a\} \cup\{f\} \succ\{b\} \cup\{f\}$. Analogously, we can conclude from the premises of this case that $\{c\} \cup\{d\} \succsim\{c\} \cup\{e\}$ (using Restricted Independence if $d \neq c \neq e$ and Averaging if $d=c$ or $e=c$ ). It then follows from transitivity that $\{c\} \cup\{d\} \succsim\{c\} \cup\{e\} \sim\{a\} \cup\{f\} \succ\{b\} \cup\{f\} \sim\{c\} \cup\{d\}$. Since this is a contradiction, we conclude that this case is impossible.

3. $\{a\} \prec\{b\}$ and $\{d\} \precsim\{e\}$. This case can also shown to be impossible, following a similar reasoning as for case 2 .

4. $\{a\} \prec\{b\}$ and $\{d\} \succsim\{e\}$. We then consider several subcases.

(i) $c=f$. Since $\{a\} \cup\{f\} \sim\{c\} \cup\{e\}$, we conclude that $\{a\} \sim\{e\}$ using Restricted Independence (if $a \neq f$ and $c \neq e$ ), Averaging (if $a=f$ and $c \neq e$ or $a \neq f$ and $c=e$ ) or trivially (if $a=f$ and $c=e$ ). By an analogous reasoning, we conclude from $\{c\} \cup\{d\} \sim\{b\} \cup\{f\}$ that $\{b\} \sim\{d\}$. Hence, we have $\{a\} \sim\{e\}$ and $\{b\} \sim\{d\}$. This implies that $\{a\} \cup\{d\} \sim\{b\} \cup\{e\}$ by Restricted Independence (if $a \neq d$ and $b \neq e$ ) or by Averaging (in all other cases).

(ii) Suppose $f \neq c, e \neq c \neq d, d \neq a \neq f$ and $e \neq b \neq f$. We first establish that there are $u$ and $v \in X^{\prime}$ such that $\{u\} \cup\{v\} \cap\{a\} \cup\{b\} \cup\{c\} \cup\{d\} \cup\{e\} \cup\{f\}=\emptyset$ and $\{a, u\} \sim\{c, v\}$. Suppose first $\{a\} \sim\{c\}$. Take then any $u \in X^{\prime} \backslash(\{a\} \cup\{b\} \cup$ $\{c\} \cup\{d\} \cup\{e\} \cup\{f\}$ ) (the existence of such a $u$ is secured by Proposition 7) and define $v=u$. We then immediately obtain $\{a, u\} \sim\{c, v\}$ by Restricted Independence. Suppose now that $\{a\} \prec\{c\}$. By Proposition 7 there is a $v \in X^{\prime}$ such that $\{v\} \prec\{a\}$. By Restricted Independence, one has $\{a, v\} \prec\{c, v\}$ and $\{c, v\} \prec\{c, a\}$ and, by transitivity, $\{a, v\} \prec\{c, v\} \prec\{a, c\}$. It follows from Richness that there is a $u \in X^{\prime}$ such that $\{a\} \cup\{u\} \sim\{c, v\}$. If $\{u, v\} \cap\{a, b, f, c, d, e\} \neq \emptyset$, one can repeat this procedure, starting with another $v$. The repetition of the procedure will be finite because the set $\{a\} \cup\{b\} \cup\{c\} \cup\{d\} \cup\{e\} \cup\{f\}$ is finite. Hence there exist $u, v \in X^{\prime} \backslash\{a\} \cup\{b\} \cup$ $\{c\} \cup\{d\} \cup\{e\} \cup\{f\}$ such that $\{a, u\} \sim\{c, v\}$. Using an analogous argument, one can obtain a similar conclusion if $\{a\} \succ\{c\}$ is assumed. Now, by Restricted Independence, one has $\{c, f, v\} \sim\{a, f, u\}$ and $\{a, f, u\} \sim\{c, e, u\}$. It follows from transitivity that $\{c, f, v\} \sim\{c, e, u\}$ so that $\{f, v\} \sim\{e, u\}$ must hold by Restricted Independence. Using Restricted Independence again, we obtain from $\{a, u\} \sim\{c, v\}$ that $\{a, d, u\} \sim$ $\{c, d, v\}$ and from $\{c, d\} \sim\{b, f\}$ that $\{c, d, v\} \sim\{b, f, v\} \sim\{b, e, u\}$. It follows from transitivity that $\{a, d, u\} \sim\{b, e, u\}$ and, by Restricted Independence, $\{a, d\} \sim\{b, e\}$.

(iii) Suppose $c \neq f, e \neq c \neq d$, and $e \neq b \neq f$. The only difference with subcase (ii) is that we relax the constraint ' $d \neq a \neq f$ '. Hence this case is more general than 
(ii). Suppose, contrary to the asserted implication of the Lemma, that $\{a\} \cup\{d\} \not$ $\{b\} \cup\{e\}$. Since $\succsim$ is complete, two symmetric cases can arise: $\{a\} \cup\{d\} \prec\{b\} \cup\{e\}$ or $\{a\} \cup\{d\} \succ\{b\} \cup\{e\}$. We only handle the first one. We first show that we can find distinct $a^{\prime}$ and $f^{\prime} \in X^{\prime} \backslash\{a\} \cup\{b\} \cup\{c\} \cup\{d\} \cup\{e\} \cup\{f\}$ such that $\left\{a^{\prime}\right\} \prec\{a\}$, $\{f\} \prec\left\{f^{\prime}\right\}$ and $\left\{a^{\prime}, f^{\prime}\right\} \sim\{a\} \cup\{f\}$. The existence of $a^{\prime} \in X^{\prime}$ such that $\left\{a^{\prime}\right\} \prec\{a\}$ is secured by Proposition 7. Assume first $a=f$. Either $\left\{a^{\prime}, g\right\} \prec\{a\}$ for all $g \in X^{\prime}$ or there exists some $g^{\prime}$ such that $\left\{a^{\prime}, g^{\prime}\right\} \succsim\{a\}$. In the second case, the existence of some $f^{\prime}$ such that $\left\{a^{\prime}, f^{\prime}\right\} \sim\{a\}$ follows from Richness. In the first case, choose by Proposition 7 some $\widehat{g} \in X^{\prime}$ such that $\{\widehat{g}\} \succ\{a\}$ and, by Certainty Equivalence, some $\widetilde{g} \in X^{\prime}$ such that $\{\widetilde{g}\} \sim\{a, \widehat{g}\}$. By Averaging and transitivity, one has $\{a\} \prec\{\widetilde{g}\} \sim\{a, \widehat{g}\} \prec\{\widetilde{g}, \widehat{g}\} \prec$ $\{\widehat{g}\}$. Hence one has $\{\widetilde{g}, \widehat{g}\} \succ\{a\} \succ\left\{a^{\prime}, \widetilde{g}\right\}$ so that, by Richness, there exists $\widetilde{a} \in X^{\prime}$ such that $\{\widetilde{a}, \widetilde{g}\} \sim\{a\}$. Choosing then $a^{\prime}=\widetilde{a}$ and $f^{\prime}=\widetilde{g}$ gives the result. If $a \neq f$, we can do the previous reasoning for the certainty equivalent of $\{a, f\}$ that exists by Certainty Equivalence. If either $a^{\prime}$ or $f^{\prime} \in\{a\} \cup\{b\} \cup\{c\} \cup\{d\} \cup\{e\} \cup\{f\}$, we can redo the procedure while starting with another $a^{\prime}$. Since the set $\{a\} \cup\{b\} \cup\{c\} \cup\{d\} \cup\{e\} \cup\{f\}$ is finite, we will redo the procedure at most a finite number of times. Hence we are sure to find $a^{\prime}$ and $f^{\prime} \in X^{\prime} \backslash\{a\} \cup\{b\} \cup\{c\} \cup\{d\} \cup\{e\} \cup\{f\}$ such that $\left\{a^{\prime}\right\} \prec\{a\}$, $\{f\} \prec\left\{f^{\prime}\right\}$ and $\left\{a^{\prime}, f^{\prime}\right\} \sim\{a\} \cup\{f\}$ and, by redoing the above procedure as many times as required, we can choose as many distinct pairs of such $a^{\prime}$ and $f^{\prime}$ as we want. Choose now some $b^{\prime}$ such that $\left\{b^{\prime}\right\} \cup\left\{f^{\prime}\right\} \sim\{b, f\}$. This is possible because $\left\{b, f^{\prime}\right\} \succ\{b, f\}$ thanks to Restricted Independence. Moreover it is impossible that $\{\widetilde{b}\} \cup\left\{f^{\prime}\right\} \succ\{b, f\}$ for all $\widetilde{b}$ in $X^{\prime}$. Indeed, since $\left\{a^{\prime}, f^{\prime}\right\} \sim\{a\} \cup\{f\}$, assuming $\{\widetilde{b}\} \cup\left\{f^{\prime}\right\} \succ\{b, f\}$ for all $\widetilde{b}$ would imply, by transitivity, that $\{a\} \cup\{f\} \succ\{b, f\}$. Yet using Averaging (if $a=f$ ) or Restricted Independence (if $a \neq f$ ), this would imply in turn that $\{a\} \succ\{b\}$, contradicting our assumption that $\{a\} \prec\{b\}$. Hence, there are consequences $\widetilde{b}$ such that $\{b, f\} \succsim\left\{\widetilde{b}, f^{\prime}\right\}$ so that, by Richness, one can find $b^{\prime}$ such that $\left\{b^{\prime}, f^{\prime}\right\} \sim\{b, f\}$. Given our flexibility for choosing $a^{\prime}$ and $f^{\prime}$ it is clear that $b^{\prime}$ can be chosen so that it does not belong to $\{a\} \cup\left\{a^{\prime}\right\} \cup\{b\} \cup\{c\} \cup\{d\} \cup\{e\} \cup\{f\} \cup\left\{f^{\prime}\right\}$. Thanks to case (ii), we know that we can obtain $\left\{a^{\prime}, d\right\} \sim\left\{b^{\prime}, e\right\}$ if we replace $a$ by $a^{\prime}, f$ by $f^{\prime}$ and $b$ by $b^{\prime}$ in the antecedent clause of the lemma. Since $\left\{a^{\prime}\right\} \prec\{a\}$, we know that $\left\{a^{\prime}, d\right\} \prec\{a\} \cup\{d\}$ by Restricted Independence (if $a \neq d$ ) or by Averaging (if $a=d$ ). Hence, it follows from transitivity that $\left\{b^{\prime}, e\right\} \sim\left\{a^{\prime}, d\right\} \prec\{a\} \cup\{d\} \prec\{b\} \cup\{e\}$. Combine now as before the axioms of Averaging and Richness to find $b^{\prime \prime}$ such that $\{a\} \cup\{d\} \prec\left\{b^{\prime \prime}\right\} \cup\{e\} \prec\{b\} \cup\{e\}$. Using Richness, one can also find some $f^{\prime \prime}$ such that $\{a\} \cup\{d\} \sim\left\{b^{\prime \prime}\right\} \cup\left\{f^{\prime \prime}\right\}$ and some $a^{\prime \prime}$ such that $\left\{a^{\prime \prime}\right\} \cup\left\{f^{\prime \prime}\right\} \sim\{f\} \cup\{e\}$. As before, we have the flexibility to find these $b^{\prime \prime}, f^{\prime \prime}$ or $a^{\prime \prime}$ in such a way that they not belong to $\{a\} \cup\{b\} \cup\{c\} \cup\{d\} \cup\{e\} \cup\{f\}$. Thanks to case (ii) again, we can obtain the conclusion that $\left\{a^{\prime \prime}, d\right\} \sim\left\{b^{\prime \prime}, e\right\}$. Since $\left\{a^{\prime \prime}\right\} \prec\{a\}$, we have $\left\{a^{\prime \prime}\right\} \cup\{d\} \prec\{a\} \cup\{d\}$ by Restricted Independence (if $a \neq d$ ) or by Averaging (if $a=d$ ). We then obtain from transitivity that $\{a\} \cup\{d\} \prec\left\{b^{\prime \prime}, e\right\} \sim\left\{a^{\prime \prime}, d\right\} \prec\{a\} \cup\{d\}$, a contradiction.

(iv) Suppose $c \neq f$ and $e \neq b \neq f$. The difference with case (iii) is that we relax the constraint ' $e \neq c \neq d$ '. Hence, as before, this case is more general than case (iii). Suppose by contradiction that the lemma is false and that $\{a\} \cup\{d\} \nsim\{b\} \cup\{e\}$. As before, the completeness of $\succsim$ implies either $\{a\} \cup\{d\} \prec\{b\} \cup\{e\}$ or $\{a\} \cup\{d\} \succ$ $\{b\} \cup\{e\}$. Since these two cases are symmetric, we only provide the argument for the first one. Using arguments analogous to those in Case (iii), one can find $c^{\prime}$ and $d^{\prime} \in X^{\prime} \backslash\{a\} \cup\{b\} \cup\{c\} \cup\{d\} \cup\{e\} \cup\{f\}$ such that $\left\{d^{\prime}\right\} \prec\{d\},\{c\} \prec\left\{c^{\prime}\right\}$ 
and $\left\{c^{\prime}, d^{\prime}\right\} \sim\{c\} \cup\{d\}$. As in case (iii) also, one can find some $e^{\prime} \in X^{\prime} \backslash\{a\} \cup$ $\{b\} \cup\left\{c, c^{\prime}\right\} \cup\left\{d, d^{\prime}\right\} \cup\{e\} \cup\{f\}$ such that $\left\{c^{\prime}, e^{\prime}\right\} \sim\{c\} \cup\{e\}$. Thanks to case (iii), we know that we can obtain $\{a\} \cup\left\{d^{\prime}\right\} \sim\{b\} \cup\left\{e^{\prime}\right\}$ out of the assumption that $\{a\} \cup\{f\} \sim\left\{c^{\prime}\right\} \cup\left\{e^{\prime}\right\}$ and $\left\{c^{\prime}\right\} \cup\left\{d^{\prime}\right\} \sim\{b\} \cup\{f\}$. Since $\left\{d^{\prime}\right\} \prec\{d\}$, we have $\left\{a, d^{\prime}\right\} \prec\{a\} \cup\{d\}$ by Restricted Independence (if $a \neq d$ ) or Averaging (if $a=d$ ). Hence by transitivity we have: $\{b\} \cup\left\{e^{\prime}\right\} \sim\{a\} \cup\left\{d^{\prime}\right\} \prec\{a\} \cup\{d\} \prec\{b\} \cup\{e\}$. Using analogous argument as for the consequences $a^{\prime \prime}, b^{\prime \prime}$ and $f^{\prime \prime}$ of case (iii), find now $c^{\prime \prime}, d^{\prime \prime}$ and $e^{\prime \prime} \in X^{\prime} \backslash\{a\} \cup\{b\} \cup\left\{c, c^{\prime}\right\} \cup\left\{d, d^{\prime}\right\} \cup\left\{e, e^{\prime}\right\} \cup\{f\}$ such that 1) $\{a\} \cup\{d\} \prec\left\{b, e^{\prime \prime}\right\} \prec$ $\{b\} \cup\{e\}, 2)\left\{c^{\prime \prime}, e^{\prime \prime}\right\} \sim\{a\} \cup\{f\}$ and 3) $\left\{c^{\prime \prime}, d^{\prime \prime}\right\} \sim\{b\} \cup\{f\}$. We know from (iii) that $\{a\} \cup\{f\} \sim\left\{c, e^{\prime \prime}\right\}$ and $\left\{c, d^{\prime \prime}\right\} \sim\{b\} \cup\{f\}$ implies $\{a\} \cup\left\{d^{\prime \prime}\right\} \sim\{b\} \cup\left\{e^{\prime \prime}\right\}$. Since $\left\{d^{\prime \prime}\right\} \prec\{d\}$, we have $\{a\} \cup\left\{d^{\prime \prime}\right\} \prec\{a\} \cup\{d\}$ by Restricted Independence (if $a \neq d$ ) or by Averaging (if $a=d$ ). Transitivity then yields $\{a\} \cup\{d\} \prec\left\{b, e^{\prime \prime}\right\} \sim\left\{a, d^{\prime \prime}\right\} \prec\{a\} \cup\{d\}$, a contradiction.

$(v)$ Suppose $c \neq f$. The difference with case $(i v)$ is that we relax the constraint ' $e \neq$ $b \neq f$. Hence this case is more general than case $(i v)$ and we handle it in an analogous fashion (conditional on $(i v)$ ) to what was done for case (iv) conditional to case (iii).

We notice that subcases $(i)$ and $(v)$ are exhaustive, conditional on Case 4.

5. $\{a\} \succ\{b\}$ and $\{d\} \precsim\{e\}$. This case is handled in the same way as Case 4 .

Another result used in the proof of Theorem 2 is the following.

Lemma 3 Let $\succsim$ be an ordering on $P\left(X^{\prime}\right)$ satisfying Averaging and Restricted Independence. Then for every (not necessarily distinct) consequences $a, b, c$, and $d \in X^{\prime},\{a\} \cup\{b\} \succsim$ $\{c\} \cup\{b\} \Leftrightarrow\{a\} \cup\{d\} \succsim\{c\} \cup\{d\}$.

Proof. We consider several cases.

1) $a \neq b, c \neq b, a \neq d$ and $c \neq d$. The result then follows immediately from Restricted Independence.

2) $a=b, c \neq b, a \neq d$ and $c \neq d$. Assume $\{a\} \cup\{b\} \succsim\{c\} \cup\{b\}$ or, equivalently under our assumption, that $\{a\} \succsim\{a, c\}$. By Averaging this statement is equivalent to $\{a\} \succsim\{c\}$ which is itself equivalent, using Restricted Independence, to $\{a, d\}=\{a\} \cup\{d\} \succsim$ $\{c, d\}=\{c\} \cup\{d\}$.

3) $a \neq b, c=b, a \neq d$ and $c \neq d$. Assume $\{a\} \cup\{b\} \succsim\{c\} \cup\{b\}$ or, equivalently under our assumption, that $\{a, b\} \succsim\{b\}$. By Averaging, this statement is equivalent to $\{a\} \succsim\{b\}$ and, by Restricted Independence, to $\{a, d\}=\{a\} \cup\{d\} \succsim\{b, d\}=\{c\} \cup\{d\}$.

4) $a \neq b, c \neq b, a=d$ and $c \neq d$. Assume $\{a\} \cup\{b\} \succsim\{c\} \cup\{b\}$ or, equivalently under our assumption, that $\{a, b\} \succsim\{c, b\}$. Using Restricted Independence, this is equivalent to $\{a\}=\{d\} \succsim\{c\}$ which, by Averaging, is equivalent to $\{d\}=\{a\} \cup\{d\} \succsim\{d, c\}=$ $\{c\} \cup\{d\}$.

5) $a \neq b, c \neq b, a \neq d$ and $c=d$. Assume $\{a\} \cup\{b\} \succsim\{c\} \cup\{b\}$ or, equivalently under our assumption, that $\{a, b\} \succsim\{c, b\}=\{d, b\}$. Using Restricted Independence, this is equivalent to $\{a\} \succsim\{d\}$ which, by Averaging, is equivalent to $\{a, d\}=\{a\} \cup\{d\} \succsim$ $\{d\}=\{c\} \cup\{d\}$. 
6) $a=b=c \neq d$. In that case reflexivity ensures that $\{a\} \cup\{b\} \succsim\{c\} \cup\{b\} \Leftrightarrow\{a\} \succsim$ $\{a\} \Leftrightarrow\{a\} \cup\{d\}=\{a, d\} \succsim\{a, d\}=\{c, d\}=\{c\} \cup\{d\}$.

All other cases are handled trivially using reflexivity.

\section{Proof of Theorem 2.}

Proof. Proposition 1 establishes that any UEU criterion satisfies Averaging and Restricted Independence. To prove the converse implication, consider the restriction of the ordering $\succsim$ to the set of all subsets of $X^{\prime}$ containing at most two consequences. Define the binary relation

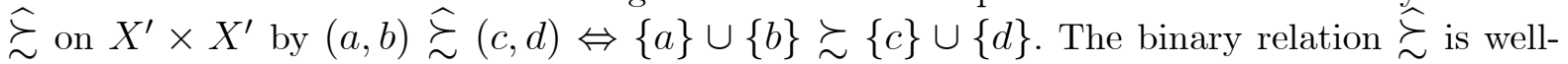
defined and is clearly an ordering of $X^{\prime} \times X^{\prime}$ if $\succsim$ is an ordering of $P\left(X^{\prime}\right)$. We also notice that, thanks to Lemma 3 , the binary relation $\widehat{\succsim}$ satisfies the property that if $(a, b) \widehat{\succsim}(c, b)$ holds for some $a, b$ and $c$, then $(a, d) \widehat{\succsim}(c, d)$ holds for all $d \in X^{\prime}$. This property is called "independence" by KLST [12] (p. 249, Definition 1). We similarly obtain that $\widehat{\succsim}$ satisfies both Thomsen's condition (see KLST, p. 250, Definition 3) and the "restricted solvability" condition (KLST, p. 250, Definition 5) using respectively Lemma 2 and the Richness axiom respectively. Finally, we note that our Archimedean axiom implies the property of the same name of KLST (p. 253, Definition 4) while our assumption that $\succsim$ is non trivial implies, thanks to Averaging (and specifically the Gardenförs condition), that each component of $X^{\prime} \times X^{\prime}$ is essential as per KLST Definition 6 (p. 256). Hence the triple $\left(X^{\prime}, X^{\prime}, \widehat{\succsim}\right)$ is an additive conjoint structure in the sense of KLST (Definition 7, p. 256). By virtue of Theorem 2 of KLST (p. 257), there are real-valued functions $\Phi_{i}$ (for $i=1,2$ ) both having $X^{\prime}$ as domain such that:

$$
(a, b) \widehat{\succsim}(c, d) \Leftrightarrow \Phi_{1}(a)+\Phi_{2}(b) \geq \Phi_{1}(c)+\Phi_{2}(d)
$$

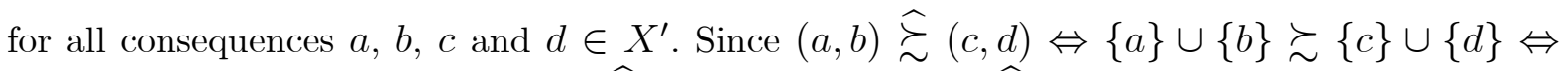
$\{b\} \cup\{a\} \succsim\{d\} \cup\{c\} \Leftrightarrow(b, a) \succsim(d, c)$, the ordering $\widehat{\succsim}$ is symmetric so that $\Phi_{1}(x)=$ $\Phi_{2}(x)=u(x)$ must hold for every $x \in X^{\prime}$ for some function $u: X^{\prime} \rightarrow \mathbb{R}$. By virtue of the second part of Theorem 2 in KLST, the function $u$ is unique up to an affine transform. Let us now show that, for all subsets $A$ and $B$ of $X^{\prime}$ containing at most two consequences, one has $A \succsim B \Leftrightarrow \sum_{a \in A} \frac{u(a)}{\# A} \geq \sum_{a \in A} \frac{u(a)}{\# A}$ so that $\succsim$ can be represented as per (1). If $\# A=\# B=1$, then one has, for all $x$ and $y \in X^{\prime},\{x\} \succsim\{y\} \Leftrightarrow\{x\} \cup\{x\} \succsim\{y\} \cup\{y\} \Leftrightarrow 2 u(x) \geq 2 u(y) \Leftrightarrow$ $u(x) \geq u(y)$ so that the numerical representation holds for that case. The argument clearly works just as well if $\# A=\# B=2$. Suppose now that $\# A=1$ and $\# B=2$. Then, for all $x$, $y$ and $z \in X^{\prime}$ such that $y \neq z$, one has:

$$
\begin{aligned}
\{x\} & \succsim\{y, z\} \Longleftrightarrow\{x\} \cup\{x\} \succsim\{y\} \cup\{z\} \\
& \Longleftrightarrow(x, x) \succsim(y, z) \\
& \Longleftrightarrow u(x)+u(x) \geq u(y)+u(z) \Longleftrightarrow u(x) \geq \frac{u(y)+u(z)}{2}
\end{aligned}
$$

so that the numerical representation holds for that case also.

\subsection{Extending Theorem 2 to all finite subsets of $\mathcal{P}\left(X^{\prime}\right)$.}

We now prove the following theorem (that restricts Theorem 1 to the set $\mathcal{P}\left(X^{\prime}\right)$ ).

Theorem 4 Let $\succsim$ be a non-trivial ordering on $\mathcal{P}\left(X^{\prime}\right)$ satisfying Richness. Then $\succsim$ satisfies Averaging, Restricted Independence and the Archimedean axiom if and only if it is a UEU criterion. Furthermore, the $u$ function in the definition of a UEU criterion is unique up to a positive affine transformation. 
The first step in proving this theorem consists in proving the following lemma 4 mentioned in section 3 .

Lemma 4 Let $U=\left\{u_{1}, \ldots, u_{n}\right\}$ be a set of $n$ numbers with arithmetic mean $\bar{u}$ such that $u_{1} \leq u_{2} \leq \ldots \leq u_{n}$. Define the $n-1$ sequences $\left\{b_{h}^{i}\right\}, i=1,2, \ldots$ and $h=1, \ldots, n-1$ by:

$$
\begin{gathered}
b_{n-1}^{0}=\left(u_{n}+u_{n-1}\right) / 2, \\
b_{h}^{0}=\left(u_{h}+b_{h+1}^{0}\right) / 2
\end{gathered}
$$

for $h=1, \ldots, n-2$ and for $i=1,2, \ldots$ :

$$
\begin{aligned}
b_{1}^{2 i-1} & =b_{1}^{2 i-2}, \\
b_{h}^{2 i-1} & =\frac{b_{h-1}^{2 i-1}+b_{h}^{2 i-2}}{2} \text { for } h=2, \ldots, n-1, \\
b_{n-1}^{2 i} & =b_{n-1}^{2 i-1} \text { and } \\
b_{h}^{2 i} & =\frac{b_{h}^{2 i-1}+b_{h+1}^{2 i}}{2} \text { for } h=1, \ldots, n-2 .
\end{aligned}
$$

Then:

$$
\lim _{i \rightarrow \infty} b_{h}^{i}=\bar{u} \text { for all } h=1, \ldots, n-1
$$

\section{Proof.}

We find useful to represent the sequence defined in this lemma in the following array, with $n-1$ columns and an infinite number of rows:

\begin{tabular}{|l|l|l|l|l|l|}
\hline & 1 & 2 & $\ldots$ & $n-2$ & $n-1$ \\
\hline $\mathbf{b}^{0}$ & $\left(u_{1}+b_{2}^{0}\right) / 2$ & $\left(u_{2}+b_{3}^{0}\right) / 2$ & $\ldots \leftarrow$ & $\left(u_{n-2}+b_{n-1}^{0}\right) / 2$ & $\left(u_{n-1}+u_{n}\right) / 2$ \\
\hline $\mathbf{b}^{1}$ & $\left(u_{1}+b_{2}^{0}\right) / 2$ & $\left(b_{1}^{1}+b_{2}^{0}\right) / 2$ & $\rightarrow \ldots$ & $\left(b_{n-3}^{1}+b_{n-2}^{0}\right) / 2$ & $\left(b_{n-2}^{1}+b_{n-1}^{0}\right) / 2$ \\
\hline $\mathbf{b}^{2}$ & $\left(b_{2}^{2}+b_{1}^{1}\right) / 2$ & $\left(b_{3}^{2}+b_{2}^{1}\right) / 2$ & $\ldots \leftarrow$ & $\left(b_{n-1}^{2}+b_{n-2}^{1}\right) / 2$ & $\left(b_{n-2}^{1}+b_{n-1}^{0}\right) / 2$ \\
\hline $\mathbf{b}^{3}$ & $\left(b_{2}^{2}+b_{1}^{1}\right) / 2$ & $\left(b_{1}^{3}+b_{2}^{2}\right) / 2$ & $\rightarrow \ldots$ & $\left(b_{n-3}^{3}+b_{n-2}^{2}\right) / 2$ & $\left(b_{n-2}^{3}+b_{n-1}^{2}\right) / 2$ \\
\hline$\ldots$ & $\ldots$ & $\ldots$ & $\ldots$ & $\ldots$ & $\ldots$ \\
\hline
\end{tabular}

We are going to show that the "grand" sequence that starts from the "north-east" of the array and follows the arrows up to infinity, converges to $\bar{u}$. Since the sequence $\left\{b_{h}^{i}\right\}$ is the $h^{\text {th }}$ column of this array and, therefore, a subsequence of the grand sequence, the conclusion of the lemma would follow immediately. Define accordingly the grand sequence $\left\{\widehat{b}^{t}\right\}$, for $t=i(n-1)+1, \ldots,(i+1)(n-1)$, and $i=0,1,2, \ldots$ by:

$$
\begin{aligned}
& \widehat{b}^{t}=b_{n-(t+1-(i(n-1)+1))}^{i} \text { if } i \text { is even and } \\
& \widehat{b}^{t}=b_{t+1-(i(n-1)+1)}^{i} \text { if } i \text { is odd }
\end{aligned}
$$

Any element of the grand sequence can be written as a weighted average of $\left\{u_{1}, \ldots, u_{n}\right\}$. In particular, for all $t=1, \ldots$, there exists $n-1$ real numbers $\beta_{1}^{t}, \ldots, \beta_{n-1}^{t}$ such that:

$$
\widehat{b}^{t}=\beta_{1}^{t} u_{1}+\beta_{2}^{t} u_{2}+\ldots .+\beta_{n-1}^{t} u_{n-1}+\beta_{n-1}^{t} u_{n}
$$

Moreover inspection reveals that $\beta_{h}^{t}$ is defined by the following recursive formula: 


$$
\begin{aligned}
\beta_{h}^{t} & =0 \text { if } t \in\{1, \ldots, n-h-1\} \\
\beta_{h}^{n-h} & =\frac{1}{2} \text { and } \\
\beta_{h}^{t} & =\frac{1}{2}\left(\beta_{h}^{t-1}+\beta_{h}^{2 m(t)-t+1}\right) \text { if } t \geq n-h+1
\end{aligned}
$$

where $m(t)$ is defined as the largest integer strictly smaller than $t$ that is divisible by $n-1$. In order to prove the Lemma, it suffices to prove that $\lim _{t \rightarrow \infty} \beta_{h}^{t}=\frac{1}{n}$ for all $h$. In what follows we will fix $h \in\{1, . ., n-1\}$ and drop the subscript $h$ from the sequence $\left\{\beta_{h}^{t}\right\}$ for notational convenience.

Once again, it is convenient to refer to the aforementioned representation of the sequence $\left\{\beta^{t}\right\}, t=1$,. as an array with $n-1$ columns and an infinite number of rows. We start from the first row with $\beta^{1}$ and move left until we reach $\beta^{n-1}$. We then move down to the second row where the first element from the left is $\beta^{n}$. Let $t$ be an arbitrary integer. If we write $t=m(t)+s$, it follows that $\beta^{t}$ lies in the $(m(t)+1)^{t h}$ row of this array. If $m(t)$ is even the $\left(\frac{m(t)}{n-1}+1\right)^{t h}$ row is increasing from right to left so that $\beta^{t}$ is the $s^{t h}$ element from the right in this row. If $m(t)$ is odd, then $\beta^{t}$ is the $s^{t h}$ element from the left in the $\left(\frac{m(t)}{n-1}+1\right)^{t h}$ row which increases from left to right. It follows that in this array, $\beta^{t}$ for $t>n-1$ is the arithmetic mean of the element which immediately precedes it in the row and the element of the same column in the row above.

The proof proceeds in two steps. The first is to show that the sequence $\left\{\beta^{t}\right\}, t=1, \ldots$ is convergent and the second is to show that the limit of the sequence is, in fact $\frac{1}{n}$. In order to establish the first step, we first record the two following properties $P 1$ and $P 2$ of the sequence which can be easily verified.

$P 1$. Let $r>1$ be an odd integer. The sequence strictly increases from $\beta^{(r-1)(n-1)+1}$ to $\beta^{(r-1)(n-1)+h}$ and then strictly decreases from $\beta^{(r-1)(n-1)+h}$ to $\beta^{r(n-1)}$. If $r$ is an even integer, then the sequence strictly increases from $\beta^{(r-1)(n-1)+1}$ to $\beta^{(r-1)(n-1)+n-h}$ and strictly decreases from $\beta^{(r-1)(n-1)+n-h}$ to $\beta^{r(n-1)}$. Thus for every row $r$ in the array, the sequence increases from the right as we move left for $h$ terms and then decreases for the remaining $n-h-1$ terms. Clearly $\beta^{(r-1)(n-1)+h}$ is the largest element of the $r^{\text {th }}$ row if $r$ is odd and $\beta^{(r-1)(n-1)+n-h}$ if $r$ is even. Note that the maximal element of any row is in the $h^{t h}$ column from the right.

$P 2$. Let $t=(n-1) r+s$ where $m(t)=r(n-1)$ (note that $1 \leq s \leq n-1)$. Then $\beta^{t}=$ $\frac{1}{2} \beta^{(n-1)(r-1)+(n-s)}+\frac{1}{2^{2}} \beta^{(n-1)(r-1)+(n-s-1)}+\ldots .+\frac{1}{2^{s-1}} \beta^{(n-1)(r-1)+(n-2)}+\frac{1}{2^{s-1}} \beta^{(n-1)(r-1)+(n-1)}$. Thus each term of the sequence can be expressed as the weighted sum of the terms of the sequence in the row above.

CLAIM: Let $r>1$ be an integer. If $r$ is odd, then :

(i) $\beta^{(r-1)(n-1)+h}-\beta^{r(n-1)}<\gamma_{1}\left[\beta^{(r-1)(n-1)+n-h}-\beta^{(r-1)(n-1)+1}\right]$ and

(ii) $\beta^{(r-1)(n-1)+h}-\beta^{(r-1)(n-1)+1}<\gamma_{2}\left[\beta^{(r-1)(n-1)+n-h}-\beta^{(r-1)(n-1)}\right]$

and if $r$ is even, then:

(iii) $\beta^{(r-1)(n-1)+n-h}-\beta^{(r-1)(n-1)+1}<\gamma_{1}\left[\beta^{(r-2)(n-1)+n-h}-\beta^{(r-1)(n-1)}\right]$ and,

(iv) $\beta^{(r-1)(n-1)+n-h}-\beta^{r(n-1)}<\gamma_{2}\left[\beta^{(r-1)(n-1)+h}-\beta^{(r-1)(n-1)+1}\right]$

where

$$
\gamma_{1}=\frac{2^{n-h-1}-1}{2^{n-h-1}}
$$


and

$$
\gamma_{2}=\frac{2^{h-1}-1}{2^{h-1}}
$$

PROOF OF THE CLAIM: We first prove (ii). We do that by first noting that, according to $P 2$ :

$$
\beta^{(r-1)(n-1)+h}=\frac{1}{2} \beta^{(r-1)(n-1)+n-h}+\ldots+\frac{1}{2^{h-1}} \beta^{(r-1)(n-1)+(n-2)}+\frac{1}{2^{h-1}} \beta^{(r-1)(n-1)+(n-1)}
$$

Since $\beta^{(r-1)(n-1)+1}=\beta^{(r-1)(n-1)}$ and $\beta^{(r-2)(n-1)+n-h}$ is the largest term in the $(r-1)^{t h}$ row according to $P 1$, we conclude that :

$$
\begin{aligned}
\beta^{(r-1)(n-1)+h}-\beta^{(r-1)(n-1)+1} & <\beta^{(r-1)(n-1)+(n-h)}\left[\frac{1}{2}+\ldots+\frac{1}{2^{h-1}}\right] \\
& -\left[1-\frac{1}{2^{h-1}}\right] \beta^{(r-1)(n-1)} \\
& =\left[1-\frac{1}{2^{h-1}}\right]\left[\beta^{(r-1)(n-1)+(n-h)}-\beta^{(r-1)(n-1)}\right]
\end{aligned}
$$

Since $\gamma_{2}=\left(1-\frac{1}{2^{h-1}}\right)$, this establishes $(i i)$.

We now prove (iii). According to P2:

$$
\beta^{(r-1)(n-1)+n-h}=\frac{1}{2} \beta^{(r-1)(n-1)+h}+\ldots+\frac{1}{2^{n-h-1}}\left[\beta^{(r-1)(n-1)+1}+\beta^{(r-1)(n-1)}\right]
$$

Since $\beta^{(r-1)(n-1)+1}=\beta^{(r-1)(n-1)}$ and since, from $P 1$, we know that $\beta^{(r-1)(n-1)+h}$ is the largest term in the $(r-1)^{t h}$ row, we obtain:

$$
\begin{aligned}
\beta^{(r-1)(n-1)+(n-h)}-\beta^{(r-1)(n-1)+1} & <\left[\frac{1}{2}+\ldots+\frac{1}{2^{n-h-1}}\right] \beta^{(r-1)(n-1)+(n-h)} \\
& -\left[1-\frac{1}{2^{n-h-1}}\right] \beta^{(r-1)(n-1)+1} \\
& =\left[1-\frac{1}{2^{n-h-1}}\right]\left[\beta^{(r-2)(n-1)+n-h}-\beta^{(r-1)(n-1)+1}\right]
\end{aligned}
$$

Since $\gamma_{1}=\left(1-\frac{1}{2^{n-h-1}}\right)$, this establishes $($ iii $)$.

We now prove $(i)$. Applying $P 2$, we have:

$$
\begin{aligned}
\beta^{(r-1)(n-1)+h} & =\frac{1}{2} \beta^{(r-1)(n-1)+n-h}+\ldots+\frac{1}{2^{h-1}} \beta^{(r-1)(n-1)+1} \\
& +\frac{1}{2^{h-1}} \beta^{(r-1)(n-1)}
\end{aligned}
$$

and:

$$
\begin{aligned}
\beta^{r(n-1)} & =\frac{1}{2} \beta^{(r-1)(n-1)+1}+\ldots+\frac{1}{2^{n-h}} \beta^{(r-1)(n-1)+n-h} \\
& +\ldots+\frac{1}{2^{n-2}} \beta^{(r-1)(n-1)+1}+\frac{1}{2^{n-2}} \beta^{(r-1)(n-1)}
\end{aligned}
$$

We thus have:

$$
\begin{gathered}
\Delta=\beta^{(r-1)(n-1)+h}-\beta^{r(n-1)} \\
=\left[\frac{1}{2}-\frac{1}{2^{n-h}}\right] \beta^{(r-1)(n-1)+n-h}+\ldots .+\left[\frac{1}{2^{h-1}}-\frac{1}{2^{n-2}}\right] \beta^{(r-1)(n-1)+1} \\
+\left[\frac{1}{2^{h-1}}-\frac{1}{2^{n-2}}\right] \beta^{(r-1)(n-1)} \\
-\frac{1}{2} \beta^{(r-1)(n-1)+1} \ldots . .-\frac{1}{2^{n-h-1}} \beta^{(r-1)(n-1)+(n-h-1)}
\end{gathered}
$$


Note that, according to $P 1, \beta^{(r-1)(n-1)+n-h}$ is the largest element in its row. This, combined with the fact that:

$$
\beta^{(r-1)(n-1)+1}<\ldots<\beta^{(r-1)(n-1)+n-h-1}
$$

implies:

$$
\begin{aligned}
\Delta & <\left[\left(\frac{1}{2}-\frac{1}{2^{n-h}}\right)+\ldots+\left(\frac{1}{2^{h-1}}-\frac{1}{2^{n-2}}\right)\right. \\
& \left.+\left(\frac{1}{2^{h-1}}-\frac{1}{2^{n-2}}\right)\right] \beta^{(r-1)(n-1)+n-h} \\
& -\left(\frac{1}{2}+\ldots .+\frac{1}{2^{n-h-1}}\right) \beta^{(r-1)(n-1)+1} \\
& =\left(\frac{1}{2}-\frac{1}{2^{n-h}}\right)\left[1+\ldots .+\frac{1}{2^{h-2}}+\frac{1}{2^{h-1}}-\frac{1}{2^{n-2}}\right] \beta^{(r-1)(n-1)+(n-h)} \\
& -\left[1-\frac{1}{2^{n-h-1}}\right] \beta^{(r-1)(n-1)+1} \\
& =\left[\left(\frac{1}{2}-\frac{1}{2^{n-h}}\right)\left(2-\frac{1}{2^{n-h}}\right)+\left(\frac{1}{2^{h-1}}-\frac{1}{2^{n-2}}\right)\right] \beta^{(r-2)(n-1)+1} \\
& -\left(1-\frac{1}{2^{n-h-1}}\right) \beta^{(r-1)(n-1)+1} \\
& =\left[1-\frac{1}{2^{n-h-1}}\right]\left[\beta^{(r-2)(n-1)+n-h}-\beta^{(r-1)(n-1)+1}\right] \\
& =\gamma_{1}\left[\beta^{(r-2)(n-1)+n-h}-\beta^{(r-1)(n-1)+1}\right]
\end{aligned}
$$

which proves $(i)$.

The proof of $(i v)$ is symmetric to that of $(i)$ and we omit the details.

We will use the inequalities in the claim to put an upper bound on the distance between terms in the same row of the array. Let $r>1$ be an odd integer. Applying $(i)$ in the claim, we have:

$$
\beta^{(r-1)(n-1)+h}-\beta^{r(n-1)}<\gamma_{1}\left(\beta^{(r-1)(n-1)+n-h}-\beta^{(r-2)(n-1)+1}\right)
$$

Observe that $\beta^{(r-2)(n-1)+n-h}-\beta^{(r-2)(n-1)+1}$ can be written as $\beta^{\left(r^{\prime}-1\right)(n-1)+n-h}-\beta^{\left(r^{\prime}-1\right)(n-1)+1}$ where $r^{\prime}=r-1$. Since $r^{\prime}$ is an even integer, we can apply (iii) to obtain:

$$
\beta^{(r-1)(n-1)+h}-\beta^{r(n-1)}<\gamma_{1}^{2}\left[\beta^{(r-3)(n-1)+n-h}-\beta^{(r-2)(n-1)+1}\right] .
$$

Hence applying $(i)$ and $(i i i)$ repeatedly, we conclude that:

$$
\begin{aligned}
\beta^{(r-1)(n-1)+h}-\beta^{r(n-1)} & <\gamma_{1}^{r-1}\left(\beta^{h}-\beta^{n-1}\right) \\
& =\gamma_{1}^{r-1}\left(\frac{1}{2}-\frac{1}{2^{n-h-1}}\right) \\
& <\frac{\gamma_{1}^{r-1}}{2} .
\end{aligned}
$$

By the same argument $\beta^{(r-1)(n-1)+n-h}-\beta^{(r-1)(n-1)+1}<\frac{\gamma_{1}^{r-1}}{2}$ when $r$ is even. Moreover, from analogous arguments, we obtain that:

$$
\beta^{(r-1)(n-1)+h}-\beta^{(r-1)(n-1)+1}<\frac{\gamma_{2}^{r-1}}{2}
$$

when $r$ is odd and:

$$
\beta^{(r-1)(n-1)+n-h}-\beta^{r(n-1)}<\frac{\gamma_{2}^{r-1}}{2}
$$

when $r$ is even. 
Let $r$ be an odd integer. The left-most and right-most terms in row $r$ are $\beta^{(r(n-1)}$ and $\beta^{(r-1)(n-1)+1}$ respectively. Using the triangle inequality and the bounds derived in the previous paragraph, it follows that:

$$
\begin{aligned}
\left\|\beta^{r(n-1)}-\beta^{(r-1)(n-1)+1}\right\| & \leq\left\|\beta^{r(n-1)}-\beta^{r(n-1)+h}\right\|+\left\|\beta^{r(n-1)+h}-\beta^{(r-1)(n-1)+1}\right\| \\
& <\frac{1}{2}\left(\gamma_{1}^{r-1}+\gamma_{2}^{r-1}\right) .
\end{aligned}
$$

If $r$ is an even integer, and the left-most and right-most terms in row $r$ are $\beta^{(r-1)(n-1)+1}$ and $\beta^{(r(n-1)}$ respectively, one has:

$$
\begin{aligned}
\left\|\beta^{r(n-1)}-\beta^{(r-1)(n-1)+1}\right\| & \leq\left\|\beta^{r(n-1)}-\beta^{r(n-1)+n-h}\right\| \\
& +\left\|\beta^{(r(n-1)+n-h}-\beta^{(r-1)(n-1)+1}\right\| \\
& <\frac{1}{2}\left(\gamma_{1}^{r-1}+\gamma_{2}^{r-1}\right) .
\end{aligned}
$$

Note that the maximal difference of terms in row $r$ is strictly less than $\frac{1}{2} \max \left[\gamma_{1}, \gamma_{2}\right]^{r-1}$.

Pick an integer $t$ such that $t=r(n-1)$ where $r$ is an odd integer i.e. $\beta^{t}$ is the left-most term in row $r$ and $m(t)=r$. Let $q=r^{\prime}(n-1)$ where $r^{\prime}>r$. Note that, by repeated application of the triangle inequality, it follows that $\left\|\beta^{t}-\beta^{q}\right\|$ is less than the sum of the differences between the left-most and right-most terms of all rows starting from $r+1$. Hence:

$$
\begin{aligned}
\left\|\beta^{t}-\beta^{q}\right\| & <\frac{1}{2}\left(\gamma_{1}^{r}+\gamma_{1}^{r+1}+\ldots . .+\gamma_{2}^{r}+\gamma_{2}^{r+1} \ldots . .\right) \\
& =\frac{1}{2}\left(\frac{\gamma_{1}^{r}}{1-\gamma_{1}}+\frac{\gamma_{2}^{r}}{1-\gamma_{2}}\right) \\
& \equiv \lambda(r) \\
& \equiv \lambda\left(\frac{m(t)}{n-1}\right)
\end{aligned}
$$

(note that we critically use the fact that $\gamma_{1}$ and $\gamma_{2}$ are strictly less than 1 ). Now let $\beta^{q}$ be a term in row $r^{\prime}$ where $r^{\prime}>r$. Applying the triangle inequality again, we have:

$$
\begin{aligned}
\left\|\beta^{t}-\beta^{q}\right\| & <\lambda(m(t))+\frac{1}{2} \max \left[\gamma_{1}, \gamma_{2}\right]^{r-1} \\
& <\lambda(m(t))+\frac{1}{2} \max \left[\gamma_{1}, \gamma_{2}\right]^{m(t)} \\
& \equiv \hat{\lambda}(t) .
\end{aligned}
$$

Observe that $\hat{\lambda}(t) \rightarrow 0$ as $t \rightarrow \infty$. Pick $\varepsilon>0$ and let $T$ be such that $\hat{\lambda}(t)<\varepsilon$ for all $t>T$. We have shown that $\left\|\beta^{T}-\beta^{q}\right\|<\varepsilon$ for all $q>T$. Hence the sequence $\beta^{t}$ is a Cauchy sequence and is convergent.

We now show that the sequence converges to $\frac{1}{n}$. Suppose it converges to $\alpha$. Let $t$ and $k$ be positive integers such that $t+1=k(n-1)$ and consider the following sequence of differences.

$$
\begin{aligned}
\beta^{t+1}-\beta^{t} & =\frac{1}{2}\left(\beta^{(k-2)(n-1)+1}-\beta^{t}\right) \\
\beta^{t}-\beta^{t-1} & =\frac{1}{2}\left(\beta^{(k-2)(n-1)+2}-\beta^{t-1}\right) \\
\ldots & =\ldots
\end{aligned}
$$




$$
\begin{aligned}
\beta^{t-(n-3)}-\beta^{t-(n-2)} & =\frac{1}{2}\left(\beta^{(k-1)(n-1)}-\beta^{(k-1)(n-1)}\right) \\
\beta^{t-(n-2)}-\beta^{t-(n-1)} & =\frac{1}{2}\left(\beta^{(k-2)(n-1)+1}-\beta^{(k-1)(n-1)-1}\right) \\
\ldots & =\ldots \\
\beta^{n-h+1}-\beta^{n-h} & =\frac{1}{2}\left(\beta^{0}-\beta^{n-h}\right)
\end{aligned}
$$

It is clear from these $t-n+h$ equalities that, except for the first $n-2$ negative terms of the right hand sides, every positive term of the first $n-1$ lines has an identical negative term in one of the lines $n+1, \ldots, 2 n$. Hence, if we sum the equalities (5)-(9), we get:

$$
\beta^{t+1}-\beta^{n-h}=-\frac{1}{2}\left(\sum_{i=1}^{n-2} \beta^{k(n-1)-i}\right)
$$

Observe that $\beta^{n-h}=1 / 2$. Also, $\left\{\beta^{k(n-1)-i}\right\}$, for $k=1, \ldots$, is a subsequence of the original sequence for all $i=1, \ldots, n-2$. Since the original sequence converges to $\alpha$, these subsequences must also converge to $\alpha$. Therefore by taking limits on both sides of the equation above, we obtain $\alpha-1 / 2=-1 / 2(n-2) \alpha$, so that $\alpha=\frac{1}{n}$, as required.

An important preliminary step in the proof of Theorem 4 is the proof that if the ordering $\succsim$ of $P\left(X^{\prime}\right)$ satisfies Restricted Independence and Averaging, then it satisfies, given Richness, the following property of Attenuation.

Definition 2 The ordering $\succsim$ of $\mathcal{P}\left(X^{\prime}\right)$ satisfies Attenuation if for all sets $A, B$ and $C \in \mathcal{P}(X)$ satisfying $A \sim B, A \cap C=B \cap C=\emptyset$ and $\# A>\# B, C \succ A$ implies $A \cup C \prec B \cup C$ and $A \succ C$ implies $A \cup C \succ B \cup C$.

The next two lemmas establish that any ordering $\succsim$ of $\mathcal{P}\left(X^{\prime}\right)$ satisfying Averaging, Restricted Independence and Richness satisfies Attenuation.

Lemma 5 Let $\succsim$ be an ordering of $\mathcal{P}(X)$ satisfying Averaging, Restricted Independence and Richness. Then, for all $A, B \in \mathcal{P}\left(X^{\prime}\right)$, such that $\# A-\# B \geq 2$, and for all sets $C \in \mathcal{P}\left(X^{\prime}\right)$ such that $C \cap(A \cup B)=\varnothing$, there are consequences $x_{1}, \ldots, x_{n} \in X^{\prime} \backslash(A \cup B \cup C)$ such that $B \sim B \cup\left\{x_{1}, \ldots, x_{n}\right\}$.

Proof. Define $n=\# A-\# B$. We distinguish three cases.

$n=2$. Using Proposition 7 and Certainty Equivalence, choose a consequence $a$ such that $B \prec$ $\{a\}$. By Averaging, $B \prec B \cup\{a\}$. Using again Proposition 7 and Certainty Equivalence, find a consequence $e \in X^{\prime}$ such that $\{e\} \succ B \cup\{a\}$. By Averaging and transitivity, we have $B \prec B \cup\{a\} \prec B \cup\{a, e\}$.

- If there is $b \in X^{\prime}$ such that $B \cup\{a, b\} \precsim B$, then, by Richness, there is a $c \in X^{\prime}$ such that $\{a, c\} \cup B \sim B$. If $a$ or $c$ belongs to $A \cup B \cup C$, we then repeat the same procedure starting with another $a$. Since $A \cup B \cup C$ is finite, we can do this repetition at most a finite number of times so that, at the end, we can find $a$ and $c \in X^{\prime}$ such that $\{a, c\} \cup B \sim B$ and $\{a, c\} \cap(A \cup B \cup C)=\emptyset$. 
- If $B \cup\{a, b\} \succ B$ for all $b \in X^{\prime}$, choose (thanks to Proposition 7 and Certainty Equivalence) $b$ and $e \in X^{\prime}$ such that $\{e\} \prec\{b\} \prec B$. By Averaging, one has $B \cup\{b, e\} \prec B \cup\{b\} \prec B$ and, by assumption, $B \cup\{a, b\} \succ B$. Hence, by Richness, there is a $c \in X^{\prime}$ such that $B \cup\{b, c\} \sim B$. If $b$ or $c$ belongs to $A \cup B \cup C$, we can repeat the same reasoning starting with another $b$. Again, the finiteness of $A \cup B \cup C$ guarantees that the repetition of the procedure will be finite and will lead, eventually, to $b$ and $c$ such that $\{b, c\} \cup B \sim B$ and $\{b, c\} \cap(A \cup B \cup C)=\emptyset$.

$n=3$. We have just proved that we can find $a$ and $c \in X^{\prime}$ such that $B \cup\{a, c\} \sim B$ and $\{a, c\} \cap(A \cup B)=\emptyset$. It can be noticed that $\{a\} \nsim\{c\}$. Choose now (thanks again to Proposition 7 and Certainty Equivalence) some $d \in X^{\prime}$ such that $\{d\} \prec B \cup\{a, c\}$. By Averaging and transitivity, one has $B \cup\{a, c, d\} \prec B \cup\{a, c\} \sim B$. Choose also (Proposition 7 and Certainty Equivalence) some $e \in X^{\prime}$ such that $\{e\} \succ B \cup\{a, c\}$. By Averaging and transitivity, $B \cup\{a, c, e\} \succ B \cup\{a, c\} \sim B$. By Richness, there is a $f \in X^{\prime}$ such that $B \cup\{a, c, f\} \sim B \cup\{a, c\} \sim B$. By Restricted Independence and transitivity, we must have either $\{a\} \succ\{f\} \succ\{c\}$ or $\{a\} \prec\{f\} \prec\{c\}$. If $f \in A \cup B \cup C$, then we can redo the procedure as many times as required starting with another $a$ or $c$.

$n>3$. If $n=2 m$ for some integer $m>1$, then we apply $m$ times the reasoning of the case $n=2$. If $n=2 m+1$ for some integer $m>1$, then we apply $(m-1)$ times the reasoning of the case $n=2$ and once the reasoning of case $n=3$.

Lemma 6 Let $\succsim$ be an ordering on $\mathcal{P}\left(X^{\prime}\right)$ satisfying Averaging, Restricted Independence and Richness. Then $\succsim$ satisfies the property of Attenuation.

Proof. Let $A$ and $B$ be sets in $\mathcal{P}(X)$ such that $A \sim B$ and $\# A>\# B$ and let $n=\# A-\# B$ and let $C$ be a set in $\mathcal{P}(X)$ such that $A \nsim C$. Since the argument works symmetrically for $A \succ C$ or $A \prec C$, we only provide it for the later case. The argument requires that we distinguish 3 cases.

$n \geq 2$. By Lemma 5 , there are $x_{1}, \ldots, x_{n}$ such that $B \cup\left\{x_{1}, \ldots, x_{n}\right\} \sim B$ and $\left\{x_{1}, \ldots, x_{n}\right\} \cap$ $(B \cup C)=\emptyset$. By Restricted Independence, $A \cup C \sim B \cup\left\{x_{1}, \ldots, x_{n}\right\} \cup C$. By Averaging, $\left\{x_{1}, \ldots, x_{n}\right\} \sim B \sim A$. Suppose $A \prec C$. By Averaging, $B \prec B \cup C$. Hence $\left\{x_{1}, \ldots, x_{n}\right\} \prec$ $B \cup C$. By Averaging again, $B \cup\left\{x_{1}, \ldots, x_{n}\right\} \cup C \prec B \cup C$. By transitivity, $A \cup C \prec B \cup C$.

$n=1, \# B \geq 2$. We first show that there exists some $x \in X^{\prime}$ and some set $B^{\prime} \in \mathcal{P}\left(X^{\prime}\right)$ such that $x \notin B^{\prime},\{x\} \sim B^{\prime} \sim B, \# B^{\prime}=\# B$ and $B^{\prime} \cap C=\emptyset$. Indeed, use Certainty Equivalence to define $x$ by $\{x\} \sim B$. If $x \notin B$, then define $B^{\prime}=B$ and the proof is done. If $x \in B$, choose a $c \in X^{\prime}$ such that $\{c\} \prec\{x\}$ and $\{c\} \succsim\{y\}$ for all $y \in B \cup C$ (if any) such that $\{y\} \prec\{x\}$. The finiteness of $B \cup C$ as well as Proposition 7 guarantees the existence of such a c. Using similar arguments, one can also find a consequence $d \in X^{\prime}$ such that $\{x\} \prec\{d\}$ and $\{d\} \precsim\{z\}$ for all $z \in B \cup C$ (if any) such that $\{x\} \prec\{z\}$. Moreover, $c$ and $d$ can be chosen in such a way that $\{x\} \precsim\{c, d\}$. Indeed, if $\{x\} \succ\{c, d\}$ for some initial choice of $c$ and $d$, then, we know from Averaging that $\{x, d\} \succ\{x\} \succ\{c, d\}$. Hence by Richness, there exists a $c^{\prime}$ such that $\left\{c^{\prime}, d\right\} \sim\{x\}$. Since $\left\{c^{\prime}, d\right\} \sim\{x\} \succ\{c, d\}$, we must have from Restricted Independence that $\left\{c^{\prime}\right\} \succ\{c\}$ and, since $\{d\} \succ\{x\}$ and $\left\{c^{\prime}, d\right\} \sim\{x\}$, it follows from Averaging and transitivity that $\{x\} \succ\left\{c^{\prime}\right\}$. We then have $\{x\} \succ\left\{c^{\prime}\right\} \succ\{c\} \succsim\{y\}$ for all $y \in B \cup C$ (if any) such that $\{y\} \prec\{x\}$. Hence replacing $c$ by $c^{\prime}$ leads immediately 
to the statement that $\{x\} \precsim\left\{c^{\prime}, d\right\}$. Assuming therefore $\{x\} \precsim\{c, d\}$, we consider two cases.

1: $\# B=2 m$, for some strictly positive integer $m$. Choose $m$ different consequences $z_{1}, \ldots, z_{m} \in X^{\prime}$ such that $\{c\} \prec\left\{z_{1}\right\} \prec \ldots \prec\left\{z_{m}\right\} \prec\{x\}$. This is clearly possible thanks to Certainty Equivalence. By assumption, $z_{i} \notin B \cup C$. For $i=1 \ldots m$, define $z_{i}^{\prime}$ by $\{x\} \sim\left\{z_{i}, z_{i}^{\prime}\right\}$. This is possible thanks to Richness and the fact that $\{x\} \precsim\{c, d\} \precsim$ $\left\{z_{i}, d\right\}$ (by Restricted Independence and transitivity) and that $\{x\} \succ\left\{z_{i}\right\} \succ\left\{z_{i}, c\right\} \succ$ $\{c\}$ (by Averaging and transitivity). Using again Averaging and transitivity, one obtains that $\left\{z_{i}, z_{i}^{\prime}\right\} \sim\{x\} \succ\left\{z_{i}, x\right\}$. It then follows from Restricted Independence that $\left\{z_{i}^{\prime}\right\} \succ$ $\{x\}$. We now prove that $\left\{z_{i}^{\prime}\right\} \prec\{d\}$, for $i=1 \ldots m$. Suppose by contradiction, using the completeness of $\succsim$, that $\{d\} \precsim\left\{z_{i}^{\prime}\right\}$ for some $i$. By Restricted Independence and transitivity, we would then have $\left\{z_{i}, d\right\} \precsim\left\{z_{i}, z_{i}^{\prime}\right\} \sim\{x\}$. Yet, since $\{c\} \prec\left\{z_{i}\right\}$, we have by Restricted Independence and transitivity that $\{c, d\} \prec\left\{z_{i}, d\right\} \precsim\left\{z_{i}, z_{i}^{\prime}\right\} \sim\{x\}$, in violation of $\{x\} \precsim\{c, d\}$. Hence, since $\{x\} \prec\left\{z_{i}^{\prime}\right\} \prec\{d\}$, we know that $z_{i}^{\prime} \notin B \cup C$. Define then $B^{\prime}=\left\{z_{1}, \ldots, z_{m}, z_{1}^{\prime}, \ldots, z_{m}^{\prime}\right\}$. By repeated application of Averaging and transitivity, one obtains that $B^{\prime} \sim\{x\} \sim B$ and, by construction, that $B^{\prime} \cap C=\emptyset$ and $x \notin B^{\prime}$.

2: $\# B=2 m+1$, for some strictly positive integer $m$. Using Certainty Equivalence, define $c^{\prime}$ and $c^{\prime \prime}$ by $\left\{c^{\prime}\right\} \sim\{c, x\}$ and $\left\{c^{\prime \prime}\right\} \sim\left\{c^{\prime}, c\right\}$. We know that $\{x\} \precsim\{c, d\}$ and, by Restricted Independence and transitivity, $\{x\} \precsim\{c, d\} \prec\left\{c^{\prime \prime}, d\right\} \prec\left\{c^{\prime}, d\right\}$. Moreover, by Averaging and transitivity, we have that $\{x\} \succ\{c, x\} \sim\left\{c^{\prime}\right\} \succ\left\{c^{\prime}, c\right\} \sim\left\{c^{\prime \prime}\right\} \succ\{c\}$. Hence, using Richness, one can define $d^{\prime}$ and $d^{\prime \prime}$ by $\left\{c^{\prime}, d^{\prime}\right\} \sim\{x\}$ and $\left\{c^{\prime \prime}, d^{\prime \prime}\right\} \sim\{x\}$. As in the case '\#B=2m', we can show that $\{d\} \succ\left\{d^{\prime \prime}\right\} \succ\left\{d^{\prime}\right\} \succ\{x\}$. Hence, $\left\{c^{\prime \prime}, c^{\prime}, d^{\prime}, d^{\prime \prime}\right\} \cap(B \cup C)=$ $\emptyset$. Since $\left\{d^{\prime}\right\} \prec\left\{d^{\prime \prime}\right\}$, we have $\{x\} \sim\left\{c^{\prime}, d^{\prime}\right\} \prec\left\{c^{\prime}, d^{\prime \prime}\right\}$ (by Restricted Independence and transitivity) and, by Averaging and transitivity, that $\{x\} \prec\left\{c^{\prime}, d^{\prime \prime}, d\right\}$. Now, by Averaging again, $\left\{c^{\prime \prime}, c^{\prime}, d^{\prime}, d^{\prime \prime}\right\} \sim\{x\}$. Hence, since $\{c\} \prec\left\{c^{\prime \prime}\right\}$, we have $\left\{c, c^{\prime}, d^{\prime}, d^{\prime \prime}\right\} \prec\{x\}$ (by Restricted Independence and transitivity). Moreover, since $\{x\} \prec\left\{d^{\prime}\right\}$, Averaging implies $\left\{c^{\prime}, c, d^{\prime \prime}\right\} \prec\{x\}$. Hence, $\left\{c^{\prime}, c, d^{\prime \prime}\right\} \prec\{x\} \prec\left\{c^{\prime}, d^{\prime \prime}, d\right\}$. By Richness, there is a $e$ such that $\{x\} \sim\left\{c^{\prime}, d^{\prime \prime}, e\right\}$. One can not have $\{c\} \succsim\{e\}$ because this would imply, using Restricted Independence and transitivity, that $\{x\} \sim\left\{c^{\prime}, d^{\prime \prime}, e\right\} \precsim\left\{c^{\prime}, d^{\prime \prime}, c\right\}$, in contradiction of $\{x\} \succ\left\{c^{\prime}, d^{\prime \prime}, c\right\}$. Analogously $\{e\} \succsim\{x\}$ can not hold because, if it did, one would have, using Restricted Independence and transitivity, that $\{x\} \sim\left\{c^{\prime}, d^{\prime \prime}, e\right\} \succsim\left\{c^{\prime}, d^{\prime \prime}, x\right\}$ and, using Averaging, that $\{x\} \succsim\left\{c^{\prime}, d^{\prime \prime}\right\}$, a contradiction. Hence, since $\succsim$ is complete, $\{c\} \prec\{e\} \prec\{x\}$. We therefore conclude that $\left\{c^{\prime}, d^{\prime \prime}, e\right\} \cap B \cup C=\emptyset$. Choose now $(m-1)$ different consequences $z_{1}, \ldots, z_{m-1} \in X^{\prime}$ in such a way that $\left\{c^{\prime}\right\} \prec\left\{z_{1}\right\} \prec \ldots \prec$ $\left\{z_{m-1}\right\} \prec\{x\}$. It is always possible to choose them different from $c^{\prime}, d^{\prime \prime}$ and $e$. For $i=1 \ldots m-1$, define as in the previous case $z_{i}^{\prime}$ by $\{x\} \sim\left\{z_{i}, z_{i}^{\prime}\right\}$. As in the previous case also, we can show that $z_{i}^{\prime}$ is such that $\{x\} \prec\left\{z_{i}^{\prime}\right\} \prec\{d\}$ for $i=1 \ldots m-1$. Define therefore $B^{\prime}$ by $B^{\prime}=\left\{c^{\prime}, d^{\prime \prime}, e, z_{1}, \ldots, z_{m-1}, z_{1}^{\prime}, \ldots, z_{m-1}^{\prime}\right\}$. By Averaging, $B^{\prime} \sim\{x\} \sim B$ and, by construction, $B^{\prime} \cap B \cup C=\emptyset$ and $x \notin B^{\prime}$.

Given the existence of the set $B^{\prime}$ and the consequence $x$ with the required property, we consider two cases.

$x \notin C$. By Averaging, $B^{\prime} \cup\{x\} \sim A$. By Restricted Independence, $B \cup C \sim B^{\prime} \cup C$ and $B^{\prime} \cup\{x\} \cup C \sim A \cup C$. Suppose $A \prec C$. Hence, $B^{\prime} \prec C$ and, by Averaging, $B^{\prime} \prec B^{\prime} \cup C \prec C$. We also have $\{x\} \prec B^{\prime} \cup C$ and, by Averaging, $\{x\} \prec B^{\prime} \cup\{x\} \cup C \prec$ $B^{\prime} \cup C$. By transitivity, $A \cup C \prec B \cup C$.

$x \in C$. We must then have that $\# C>1$, as assuming otherwise would imply that 
$C=\{x\} \sim B \sim A$. Using the same argument as above, there is a set $C^{\prime} \in \mathcal{P}(X)$ satisfying $C^{\prime} \sim C, \# C^{\prime}=\# C, x \notin C^{\prime}, B \cap C^{\prime}=\emptyset, B^{\prime} \cap C^{\prime}=\emptyset, A \cap C^{\prime}=\emptyset$. By Restricted Independence, $B \cup C \sim B^{\prime} \cup C, B^{\prime} \cup C \sim B^{\prime} \cup C^{\prime}, A \cup C \sim A \cup C^{\prime}$, and $B^{\prime} \cup\{x\} \cup C^{\prime} \sim A \cup C^{\prime}$. Suppose $A \prec C$. Hence, $B^{\prime} \prec C^{\prime}$ and, by Averaging, $B^{\prime} \prec B^{\prime} \cup C^{\prime} \prec C^{\prime}$. We also have $\{x\} \prec B^{\prime} \cup\{x\} \cup C^{\prime} \prec B^{\prime} \cup C^{\prime}$. By transitivity, $A \cup C \prec B \cup C$.

$n=1, \# B=1$. Suppose first that $\# C=1$. Write $A, B$ and $C$ as: $A=\{a, b\}, B=\{x\}$ and $C=\{c\}$ and assume that $\{x\} \sim\{a, b\} \prec\{c\}$. Assume, contrary to what is required by Attenuation, that $\{a, b, c\} \succsim\{x, c\}$. By Certainty Equivalence, there exists a $z \in X^{\prime}$ such that $\{z\} \sim\{x, c\}$. Since $\{x\} \prec\{c\},\{x\} \prec\{z\} \prec\{c\}$ by Averaging so that $z$ is distinct from either $x$ or $c$. We therefore have (using Averaging and transitivity) $\{a, b, c\} \succsim\{x, c\} \sim\{z\} \sim\{x, c, z\}$. It then follows from Restricted Independence and transitivity that $\{a, b\} \succsim\{x, z\} \succ\{x\}$, contradicting $\{x\} \sim\{a, b\}$. Suppose now that $\# C>1$. Suppose $\{x\} \sim\{a, b\} \prec C$ but, contrary to what is required by Attenuation, $\{a, b\} \cup C \succsim\{x\} \cup C$. By Certainty Equivalence, there is a consequence $z \in X^{\prime}$ such $\{z\} \sim\{x\} \cup C$. By Averaging (since $x \notin C$ ), one has $\{x\} \prec\{z\} \prec C$. One has therefore $\{a, b\} \cup C \succsim\{x\} \cup C \sim\{z\}$. If $z \notin C$, then Averaging and transitivity entails that $\{z\} \sim\{x, z\} \cup C$. Using then Restricted Independence and transitivity, one obtains that $\{a, b\} \succsim\{x, z\} \succ\{x\}$, a contradiction. If $z \in C$, then apply Certainty Equivalence recursively to find a sequence of $z_{t}$ such that $\left\{z_{t}\right\} \sim\left\{z_{t-1}, x\right\}$ for $t=1, \ldots$ starting with $z_{0}=z$. Since there are only finitely many elements in $C$, one will eventually find some $t$ for which $z_{t} \notin C$ and $\{x\} \prec\left\{z_{t}\right\} \prec \ldots \prec\{z\} \prec C$. By Averaging $\{x\} \cup C \sim\{x\} \cup C \backslash\{z\} \sim\{z\}$. Since $\{z\} \succ\left\{z_{t}\right\}$, we have, by transitivity and Averaging, that $\{z\} \sim\{x\} \cup C \backslash\{z\} \succ$ $\left\{x, z_{t}\right\} \cup C \succ\left\{z_{t}\right\}$. We therefore have $\{a, b\} \cup C \succsim\{x\} \cup C \sim\{z\} \sim\{x\} \cup C \succ\left\{x, z_{t}\right\} \cup C$ which implies, thanks to Restricted Independence and transitivity, that $\{a, b\} \succ\left\{z_{t}, x\right\}$ and, by Averaging and transitivity, that $\{a, b\} \succ\{x\}$, again a contradiction.

We next establish some further auxiliary lemmas.

Lemma 7 Let $\succsim$ be an ordering on $\mathcal{P}\left(X^{\prime}\right)$ satisfying Averaging, Restricted Independence, and Richness. Then, if $A, B \in \mathcal{P}\left(X^{\prime}\right)$ and $c$ is a consequence in $X^{\prime}$ such that $A \prec B \cup\{c\}$, then there exists some $e \in X^{\prime}$ such that $\{e\} \prec\{c\}$ and $A \prec B \cup\{e\}$. Dually, if $A, B \in \mathcal{P}\left(X^{\prime}\right)$ and $c$ is a consequence in $X^{\prime}$ such that $A \succ B \cup\{c\}$, then there exists $e \in X^{\prime}$ such that $\{e\} \succ\{c\}$ and $A \succ B \cup\{e\}$.

Proof. We only prove the first statement and distinguish three cases.

(a) $A \prec B \cup\{d\}$, in which case the proof is done.

(b) $A \sim B \cup\{d\}$. Then, by Certainty Equivalence, there exists $e$ such that $\{e\} \sim\{d, c\}$. By Averaging, $\{d\} \prec\{e\} \prec\{c\}$. By Restricted Independence, $B \cup\{d\} \prec B \cup\{e\}$ so that the statement $A \prec B \cup\{e\}$ follows.

(c) $A \succ B \cup\{d\}$. In that case the Richness axiom applies and there is a consequence $f$ such that $A \sim B \cup\{f\}$ and we proceed as in case (b).

We next establish that if $\succsim$ is an ordering of $\mathcal{P}\left(X^{\prime}\right)$ satisfying Averaging, Restricted Independence, Richness and Attenuation (Lemma 6), then it satisfies the following condition. 
Condition 3 For all distinct consequences $a, b, c$ and $d \in X$ and every set $B \in \mathcal{P}(X)$ such that $\{b\} \sim\{c, d\}$ and $B \cap\{b, c, d\}=\emptyset$, we must have:

(i) $\{a\} \succsim B \cup\{b\}$ and $\{b\} \succsim\{a\}$ with at least one strict ranking imply $\{a, b\} \succ B \cup\{c, d\}$, and (ii) $\{a\} \precsim B \cup\{b\}$ and $\{b\} \prec\{a\}$ with at least one strict ranking imply $\{a, b\} \prec B \cup\{c, d\}$.

Three auxiliary lemmas are needed in order to establish this. The first of them is the following.

Lemma 8 Let $\succsim$ be an ordering on $\mathcal{P}\left(X^{\prime}\right)$ satisfying Averaging, Restricted Independence and Richness. Let $A$ and $B$ be two finite subsets of $X^{\prime}$ and let $a, b, c$ and $d$ be consequences in $X^{\prime}$ satisfying $A \cup\{a\} \sim B \cup\{b\}, \# A=\# B,\{b\} \sim\{c, d\}, a \neq b, c \neq d,\{a, b\} \cap A=\{c, d\} \cap B=\emptyset$ and $b \notin B$. Then $A \cup\{a, b\} \sim B \cup\{c, d\}$.

Proof. Suppose first $\{c\} \sim\{d\}$. By Averaging, $\{b\} \sim\{c\} \sim\{d\}$. Since $c \neq d$, we have $c \neq b$ or $d \neq b$. Assume without loss of generality that $c \neq b$. By Restricted Independence, $B \cup\{b\} \sim B \cup\{c\}$. Therefore $A \cup\{a\} \sim B \cup\{b\} \sim B \cup\{c\}$ and, by Restricted Independence, $A \cup\{a, b\} \sim B \cup\{c, b\}$. By Restricted Independence again, $B \cup\{c, b\} \sim B \cup\{c, d\}$. Finally, by transitivity, $A \cup\{a, b\} \sim B \cup\{c, d\}$.

Suppose now $\{c\} \not\{d\}$ and assume, without loss of generality, that $\{c\} \prec\{d\}$. Two cases need to be considered.

1. Assume by contradiction that $A \cup\{a, b\} \prec B \cup\{c, d\}$. Let us show that there is a $\underline{d}$ such that $A \cup\{a, b\} \prec B \cup\{c, \underline{d}\} \prec B \cup\{c, d\}$. Choose some $u$ distinct from $c$ such that $\{u\} \prec\{d\}$. The existence of such a $u$ is guaranteed by the fact that $\{c\} \prec\{d\}$ and, using Certainty Equivalence, that one can always define $u$ by $\{u\} \sim\{c, d\}$. By Averaging, one must have $\{c\} \prec\{u\} \prec\{d\}$ which, given the reflexivity of $\succsim$, implies that $u$ is distinct from both $c$ and $d$. By Restricted Independence, one has $B \cup\{c, u\} \prec B \cup\{c, d\}$. Two mutually exclusive cases can occur.

- $B \cup\{c, u\} \precsim A \cup\{a, b\}$. By Averaging and Certainty Equivalence, one can find some $e$ such that $A \cup\{a, b\} \prec e \prec B \cup\{c, d\}$. By Richness, there is $\underline{d}$ such that $B \cup\{c, \underline{d}\} \sim\{e\}$. Hence $A \cup\{a, b\} \prec B \cup\{c, \underline{d}\} \prec B \cup\{c, d\}$

- $A \cup\{a, b\} \prec B \cup\{c, u\}$. In this case, let $\underline{d}=u$.

By Certainty Equivalence, there is a $\underline{b}$ such that $\{\underline{b}\} \sim\{c, \underline{d}\}$. Notice that we can always choose $\underline{d}$ so that $\underline{d}$ and $\underline{b}$ do not belong to $B \cup\{c\} \cup A \cup\{a\}$. By Restricted Independence, $\{\underline{b}\} \prec\{b\}$. By Averaging, $\{\underline{b}\} \sim\{c, \underline{d}\} \prec\{b, \underline{b}\} \prec\{b\}$. By Restricted Independence, $B \cup\{c, \underline{d}\} \prec B \cup\{b, \underline{b}\}$. By Restricted Independence, $A \cup\{a, \underline{b}\} \prec A \cup\{a, b\}$ and $A \cup\{a, \underline{b}\} \sim B \cup\{b, \underline{b}\}$. By transitivity, $B \cup\{c, \underline{d}\} \prec A \cup\{a, b\}$. But we have previously shown that $A \cup\{a, b\} \prec B \cup\{c, \underline{d}\}$. A contradiction.

2. Assume by contradiction that $A \cup\{a, b\} \succ B \cup\{c, d\}$. This case is treated like the previous one.

The next Lemma provides the second step in the proof that Averaging, Restricted Independence and Richness imply Condition 3. 
Lemma 9 Let $\succsim$ be an ordering on $\mathcal{P}\left(X^{\prime}\right)$ satisfying Averaging, Restricted Independence and Richness and let $a, b, c$ and $d$ be consequences in $X^{\prime}$ and $B$ be a finite subset of $X^{\prime}$ such that $\{a\} \succsim B \cup\{b\},\{b\} \sim\{c, d\},\{b\} \succ\{a\}, b \notin B$ and $\{c, d\} \cap B=\emptyset$. Then there exists a finite subset $A^{\prime}$ of $X^{\prime}$ and some consequence $a^{\prime} \in X^{\prime}$ such that $A^{\prime} \cup\left\{a^{\prime}\right\} \sim B \cup\{b\}, a^{\prime} \notin A^{\prime}$ and $\# A^{\prime}=\# B$.

Proof. Start with $\{b\} \succ\{a\} \succsim B \cup\{b\}$. By Averaging, $\{b\} \succ B$. Write $B$ as $B=$ $\left\{b_{1}, \ldots, b_{r}\right\}$ with $\left\{b_{1}\right\} \precsim\left\{b_{2}\right\} \precsim \ldots \precsim\left\{b_{r}\right\}$. Let $b_{j}$ be such that $\left\{b_{j}\right\} \prec\{b\}$ and $\{b\} \precsim b_{i}$ for all $i>j$. The existence of such a $b_{j}$ is guaranteed by Averaging. By Certainty Equivalence, one can find some $b_{j}^{\prime}$ in $X^{\prime}$ such that $b_{j}^{\prime} \sim\left\{b, b_{j}\right\}$. By Averaging, $b_{j} \prec b_{j}^{\prime} \prec b$. Define $A^{\prime}$ by $A^{\prime}=B \cup\left\{b_{j}^{\prime}\right\} \backslash\left\{b_{j}\right\}$. By Averaging and transitivity, one has $A^{\prime} \succ B$. By Restricted Independence, $A^{\prime} \cup\{b\} \succ B \cup\{b\}$. By construction, $A^{\prime} \cup\left\{b_{j}\right\}=B \cup\left\{b_{j}^{\prime}\right\}$. By Restricted Independence, $B \cup\{b\} \succ B \cup\left\{b_{j}^{\prime}\right\}$. Hence $A^{\prime} \cup\{b\} \succ B \cup\{b\} \succ B \cup\left\{b_{j}^{\prime}\right\}=A^{\prime} \cup\left\{b_{j}\right\}$. By Richness, there exists some $a^{\prime}$ such that $A^{\prime} \cup\left\{a^{\prime}\right\} \sim B \cup\{b\}$. By Restricted Independence, one has $\{b\} \succ\left\{a^{\prime}\right\} \succ\left\{b_{j}^{\prime}\right\}$, which, given the definition of $A^{\prime}$, establishes that $a^{\prime} \notin A^{\prime}$.

Combining these two lemmas, we can establish the following.

Lemma 10 Let $\succsim$ be an ordering on $\mathcal{P}\left(X^{\prime}\right)$ satisfying Averaging, Restricted Independence and Richness. Then $\succsim$ satisfies Condition 3 .

Proof. We prove only part $(i)$ of condition 3, the proof of the other part being similar. Suppose that we have $\{a\} \succsim B \cup\{b\},\{b\} \sim\{c, d\},\{b\} \succ\{a\}, b \notin B$ and $\{c, d\} \cap B=\emptyset$ for consequences $a, b, c, d$ in $X^{\prime}$ and some finite subset $B$ of $X^{\prime}$. By Lemma 9, there exists a finite set $A^{\prime}$ and a consequence $a^{\prime}$ such that $A^{\prime} \cup\left\{a^{\prime}\right\} \sim B \cup\{b\}, a^{\prime} \notin A^{\prime}$ and $\# A^{\prime}=\# B$. By Lemma 8 , we must have $A^{\prime} \cup\left\{a^{\prime}, b\right\} \sim B \cup\{c, d\}$. By Certainty Equivalence, there exists some $a^{\prime \prime}$ such that $a^{\prime \prime} \sim A^{\prime} \cup\left\{a^{\prime}\right\}$. By transitivity, $\{b\} \succ\{a\} \succsim A^{\prime} \cup\left\{a^{\prime}\right\} \sim\left\{a^{\prime \prime}\right\}$. Since, by Lemma 6 , the ordering $\succsim$ satisfies Attenuation, one has $A^{\prime} \cup\left\{a^{\prime}, b\right\} \prec\left\{a^{\prime \prime}, b\right\}$. By transitivity, $\left\{a^{\prime \prime}, b\right\} \succ B \cup\{c, d\}$. Restricted Independence and $\{a\} \succsim\left\{a^{\prime \prime}\right\}$ imply $\{b, a\} \succsim\left\{b, a^{\prime \prime}\right\}$. Transitivity finally yields $\{a, b\} \succ B \cup\{c, d\}$.

We are now equipped to prove Theorem 4.

\section{Proof of Theorem 4}

Proof. Using Theorem 2, we find a function $u$ that uniquely represents (up to an affine transform) $\succsim$ as per (1) on the subset of $\mathcal{P}\left(X^{\prime}\right)$ containing sets of cardinality no greater than 2 . We want to prove that the same function $u$ can also be used to represent $\succsim$ on the whole set $\mathcal{P}\left(X^{\prime}\right)$. We must prove specifically that, for any $A \in \mathcal{P}\left(X^{\prime}\right)$ and $g \in X^{\prime}$,

$$
A \succsim\{g\} \Longleftrightarrow \sum_{a \in A} \frac{u(a)}{\# A} \geq u(g) .
$$

where $u$ is the (unique up to an affine transform) utility function identified in Theorem 2. Since $\succsim$ is complete, it is sufficient to prove $\Rightarrow$. Suppose $\# A=m$ and write $A=\left\{a_{1}, a_{2}, \ldots, a_{m}\right\}$ with $\left\{a_{1}\right\} \precsim \ldots \precsim\left\{a_{m}\right\}$. By Certainty Equivalence, there exists $b_{m-1}^{0} \in X^{\prime}$ such that $b_{m-1}^{0} \sim$ $\left\{a_{m-1}, a_{m}\right\}$. Similarly, for $i=m-2, \ldots, 1$, we can find, by Certainty Equivalence, a consequence $b_{i}^{0}$ such that $b_{i}^{0} \sim\left\{a_{i}, b_{i+1}^{0}\right\}$. Using Certainty Equivalence repeatedly, one can define this way for $j=1,2,3, \ldots$ the sequence of $b_{i}^{j}$ by:

$$
b_{1}^{2 j-1}=b_{1}^{2 j-2},
$$




$$
b_{i}^{2 j-1} \sim\left\{b_{i-1}^{2 j-1}, b_{i}^{2 j-2}\right\}
$$

for $i=2, \ldots, m-1$,

$$
b_{m-1}^{2 j}=b_{m-1}^{2 j-1}
$$

and

$$
b_{i}^{2 j} \sim\left\{b_{i}^{2 j-1}, b_{i+1}^{2 j}\right\}
$$

for $i=m-2, \ldots, 1$. We first show that:

(i) $\left\{b_{1}^{j}\right\} \precsim\left\{b_{2}^{j}\right\} \precsim \ldots \precsim\left\{b_{m-1}^{j}\right\}$,

(ii) $\left\{a_{1}\right\} \precsim\left\{b_{1}^{i}\right\} \precsim\left\{b_{1}^{i+1}\right\} \precsim\left\{b_{m-1}^{i+1}\right\} \precsim\left\{b_{m-1}^{i}\right\} \precsim\left\{a_{m}\right\}$ and

(iii) $\left\{b_{1}^{i}\right\} \precsim A \precsim\left\{b_{m-1}^{i}\right\}$.

If $\left\{a_{1}\right\} \sim\left\{a_{m}\right\}$, then, by Averaging, $\left\{a_{1}\right\} \sim A,\left\{b_{j}^{i}\right\} \sim\left\{a_{1}\right\} \sim A$ for all $i \in \mathbb{N}$ and $j \in$ $\{1, \ldots, m-1\}$ and the implications $(i)$-(iii) are immediately established. If $\left\{a_{1}\right\} \prec\left\{a_{m}\right\}$, let $k$ be the largest integer such that $\left\{a_{k}\right\} \prec\left\{a_{k+1}\right\}$. We first prove implications $(i)$ and $(i i)$. By Averaging, $\left\{a_{m-1}\right\} \precsim\left\{b_{m-1}^{0}\right\} \precsim\left\{a_{m}\right\}$. By transitivity, $\left\{a_{m-2}\right\} \precsim\left\{b_{m-1}^{0}\right\}$. By Averaging again, $\left\{a_{m-2}\right\} \precsim\left\{b_{m-2}^{0}\right\} \precsim\left\{b_{m-1}^{0}\right\}$. By repeated use of transitivity and Averaging, one is led to the conclusion that $\left\{a_{k+1}\right\} \precsim\left\{b_{k+1}^{0}\right\} \precsim\left\{b_{k+2}^{0}\right\}$. Now, by transitivity $\left\{a_{k}\right\} \prec\left\{b_{k+1}^{0}\right\}$ and, by Averaging, $\left\{a_{k}\right\} \prec\left\{b_{k}^{0}\right\} \prec\left\{b_{k+1}^{0}\right\}$. Analogously, a repeated combination of Averaging and transitivity leads to the conclusion that $\left\{a_{1}\right\} \prec\left\{b_{1}^{0}\right\} \prec\left\{b_{2}^{0}\right\}$. Hence, we have established that $\left\{a_{1}\right\} \prec\left\{b_{1}^{0}\right\} \prec\left\{b_{k+1}^{0}\right\} \precsim\left\{b_{k+2}^{0}\right\} \precsim \ldots \precsim\left\{b_{m-1}^{0}\right\} \precsim\left\{a_{m}\right\}$. Now, by Averaging, $\left\{b_{1}^{0}\right\} \prec\left\{b_{2}^{1}\right\} \prec$ $\left\{b_{2}^{0}\right\}$ and, by transitivity, $\left\{b_{2}^{1}\right\} \prec\left\{b_{3}^{0}\right\}$. Combining in this way Averaging and transitivity leads us to $\left\{b_{m-2}^{1}\right\} \prec\left\{b_{m-1}^{1}\right\} \prec\left\{b_{m-1}^{0}\right\}$ and, therefore, to $\left\{a_{1}\right\} \prec\left\{b_{1}^{0}\right\} \sim\left\{b_{1}^{1}\right\} \prec\left\{b_{2}^{1}\right\} \prec \ldots \prec$ $\left\{b_{m-1}^{1}\right\} \prec\left\{b_{m-1}^{0}\right\} \precsim\left\{a_{m}\right\}$. Repeatedly using the same reasoning, we find that, for all $i$, $\left\{b_{1}^{i}\right\} \prec\left\{b_{2}^{i}\right\} \prec \ldots \prec\left\{b_{m-1}^{i}\right\}$ and $\left\{a_{1}\right\} \precsim\left\{b_{1}^{i}\right\} \precsim\left\{b_{1}^{i+1}\right\} \precsim\left\{b_{m-1}^{i+1}\right\} \precsim\left\{b_{m-1}^{i}\right\} \precsim\left\{a_{m}\right\}$. We now turn to implication (iii) that we prove in the following steps.

Step 1. We notice that by virtue of the Gärdenfors principle, $\left\{b_{m-1}^{0}\right\} \succ A$.

Step 2. We prove that $\left\{b_{1}^{0}\right\} \prec A$. Since by assumption $a_{l}=a_{l+1}$ for all $l=k+1, \ldots, m-1$, we have by Averaging that $\left\{b_{m-1}^{0}\right\} \sim\left\{a_{m}\right\} \sim\left\{a_{m-1}, a_{m}\right\} \sim\left\{a_{m-2}\right\} \sim\left\{a_{m-2}, a_{m-1}, a_{m}\right\} \sim$ $\ldots \sim\left\{a_{k+1}, \ldots, a_{m-1}, a_{m}\right\}$. We therefore have $\left\{b_{k+1}^{0}\right\} \sim\left\{a_{k+1}, \ldots, a_{m-1}, a_{m}\right\} \sim\left\{a_{k+1}\right\}$. Now, since $\left\{a_{k}\right\} \prec\left\{b_{k+1}^{0}\right\} \sim\left\{a_{k+1}, \ldots, a_{m-1}, a_{m}\right\}$, it follows from Attenuation (satisfied thanks to Lemma 6$)$ that $\left\{a_{k}, b_{k+1}^{0}\right\} \prec\left\{a_{k}, a_{k+1}, \ldots, a_{m-1}, a_{m}\right\}$ and, since $\left\{b_{k}^{0}\right\} \sim$ $\left\{a_{k}, b_{k+1}^{0}\right\}$ and $\succsim$ is transitive, that $\left\{b_{k}^{0}\right\} \prec\left\{a_{k}, \ldots, a_{m-1}, a_{m}\right\}$. Applying the same reasoning below $k$ enables us to reach the conclusion that $\left\{b_{1}^{0}\right\} \prec\left\{a_{1}, \ldots, a_{2}, a_{m}\right\}=A$.

Step 3. Since $b_{1}^{1}=b_{1}^{0}$, we trivially have that $\left\{b_{1}^{1}\right\} \prec A$.

Step 4. We prove that $\left\{b_{m-1}^{1}\right\} \succ A$. Notice that $\left\{b_{1}^{1}\right\} \sim\left\{a_{1}, b_{2}^{0}\right\},\left\{b_{2}^{1}\right\} \sim\left\{b_{1}^{1}, b_{2}^{0}\right\},\left\{b_{2}^{0}\right\} \sim$ $\left\{a_{2}, b_{3}^{0}\right\},\left\{b_{1}^{1}\right\} \prec\left\{b_{2}^{0}\right\}$ and clause $(i)$ of Condition 3 (satisfied thanks to Lemma 10) imply that $\left\{b_{2}^{1}\right\} \succ\left\{a_{1}, a_{2}, b_{3}^{0}\right\}$. Similarly, $\left\{b_{2}^{1}\right\} \sim\left\{b_{1}^{1}, b_{2}^{0}\right\},\left\{b_{3}^{1}\right\} \sim\left\{b_{2}^{1}, b_{3}^{0}\right\}$ and clause $(i)$ of the condition 3 imply that $\left\{b_{3}^{1}\right\} \succ\left\{a_{1}, a_{2}, a_{3}, b_{4}^{0}\right\}$. Repeating this reasoning, we obtain $\left\{b_{m-2}^{1}\right\} \succ$ $\left\{a_{1}, \ldots, a_{m-2}, b_{m-1}^{0}\right\}$ and, finally, $\left\{b_{m-1}^{1}\right\} \succ\left\{a_{1}, \ldots, a_{m}\right\}=A$.

Step 5. Trivially, $\left\{b_{m-1}^{2}\right\}=\left\{b_{m-1}^{1}\right\} \succ A$. 
Step 6. We prove that $\left\{b_{1}^{2}\right\} \prec A$. We have $\left\{b_{m-1}^{2}\right\} \sim\left\{b_{m-1}^{0}, b_{m-2}^{0}\right\},\left\{b_{m-2}^{2}\right\} \sim\left\{b_{m-1}^{2}, b_{m-2}^{1}\right\}$, $\left\{b_{m-2}^{1}\right\} \sim\left\{b_{m-3}^{1}, b_{m-2}^{0}\right\}$ and $\left\{b_{m-2}^{1}\right\} \prec\left\{b_{m-1}^{2}\right\}$. Hence, by clause (ii) of condition $3,\left\{b_{m-2}^{2}\right\} \prec$ $\left\{b_{m-3}^{1}, b_{m-2}^{0}, b_{m-1}^{0}\right\}$. We also have $\left\{b_{m-2}^{2}\right\} \prec\left\{b_{m-3}^{1}, b_{m-2}^{0}, b_{m-1}^{0}\right\},\left\{b_{m-3}^{2}\right\} \sim\left\{b_{m-2}^{2}, b_{m-3}^{1}\right\}$, $\left\{b_{m-3}^{1}\right\} \sim\left\{b_{m-4}^{1}, b_{m-3}^{0}\right\}$ and $\left\{b_{m-3}^{1}\right\} \prec\left\{b_{m-2}^{2}\right\}$. Hence, by clause (ii) of Condition 3:

$\left\{b_{m-3}^{2}\right\} \prec\left\{b_{m-4}^{1}, b_{m-3}^{0}, b_{m-2}^{0}, b_{m-1}^{0}\right\}$. This process can be repeated until we obtain:

$$
\left\{b_{2}^{2}\right\} \prec\left\{b_{1}^{1}, b_{2}^{0}, b_{3}^{0}, \ldots, b_{m-1}^{0}\right\}=\left\{b_{1}^{0}, b_{2}^{0}, b_{3}^{0}, \ldots, b_{m-1}^{0}\right\} .
$$

By Lemma 7, there exists $\left\{c_{2}^{0}\right\} \prec\left\{b_{2}^{0}\right\}$ such that $\left\{b_{2}^{2}\right\} \prec\left\{b_{1}^{0}, c_{2}^{0}, b_{3}^{0}, \ldots, b_{m-1}^{0}\right\}$. Repeatedly applying Lemma 7 , we find $\left\{c_{i}^{0}\right\} \prec\left\{b_{i}^{0}\right\}$, for $i=3 \ldots m-1$ such that $\left\{b_{2}^{2}\right\} \prec\left\{b_{1}^{0}, c_{2}^{0}, c_{3}^{0}, \ldots, c_{m-1}^{0}\right\}$. This, combined with $\left\{b_{1}^{2}\right\} \sim\left\{b_{2}^{2}, b_{1}^{1}\right\},\left\{b_{1}^{1}\right\} \sim\left\{a_{1}, b_{2}^{0}\right\},\left\{b_{1}^{1}\right\} \prec\left\{b_{2}^{2}\right\}$ and clause (ii) of Condition 3, implies:

$\left\{b_{1}^{2}\right\} \prec\left\{a_{1}, c_{2}^{0}, b_{2}^{0}, c_{3}^{0}, \ldots, c_{m-1}^{0}\right\}$. By Averaging, it follows that $\left\{c_{2}^{0}, b_{2}^{0}\right\} \prec\left\{b_{2}^{0}\right\} \sim\left\{a_{2}, b_{3}^{0}\right\}$. By Restricted Independence, one has $\left\{b_{1}^{2}\right\} \prec\left\{a_{1}, a_{2}, c_{3}^{0}, b_{3}^{0}, \ldots, c_{m-1}^{0}\right\}$. By Averaging, $\left\{c_{3}^{0}, b_{3}^{0}\right\} \prec$ $\left\{b_{3}^{0}\right\} \sim\left\{a_{3}, b_{4}^{0}\right\}$. By Restricted Independence:

$$
\left\{b_{1}^{2}\right\} \prec\left\{a_{1}, a_{2}, a_{3}, c_{4}^{0}, b_{4}^{0}, \ldots, c_{m-1}^{0}\right\} .
$$

Repeating this process leads us to the conclusion that:

$$
\left\{b_{1}^{2}\right\} \prec\left\{a_{1}, a_{2}, \ldots, a_{m-2}, c_{m-1}^{0}, b_{m-1}^{0}\right\} .
$$

By Averaging, $\left\{c_{m-1}^{0}, b_{m-1}^{0}\right\} \prec\left\{b_{m-1}^{0}\right\} \sim\left\{a_{m-1}, a_{m}\right\}$. By Restricted Independence:

$$
\left\{b_{1}^{2}\right\} \prec\left\{a_{1}, a_{2}, \ldots, a_{m-2}, a_{m-1}, a_{m}\right\}=A .
$$

Step 7. Trivially, $\left\{b_{1}^{3}\right\}=\left\{b_{1}^{2}\right\} \prec A$.

Step 8. We prove that $\left\{b_{m-1}^{3}\right\} \succ A$. We have $\left\{b_{1}^{3}\right\} \sim\left\{b_{1}^{1}, b_{2}^{2}\right\},\left\{b_{2}^{3}\right\} \sim\left\{b_{1}^{3}, b_{2}^{2}\right\},\left\{b_{2}^{2}\right\} \sim$ $\left\{b_{2}^{1}, b_{3}^{2}\right\}$ and $\left\{b_{2}^{2}\right\} \succ\left\{b_{1}^{3}\right\}$. Hence, by clause $(i)$ of Condition $3,\left\{b_{2}^{3}\right\} \succ\left\{b_{1}^{1}, b_{2}^{1}, b_{3}^{2}\right\}$. We also have $\left\{b_{3}^{3}\right\} \sim\left\{b_{2}^{3}, b_{3}^{2}\right\},\left\{b_{3}^{2}\right\} \sim\left\{b_{3}^{1}, b_{4}^{2}\right\}$ and $\left\{b_{3}^{2}\right\} \succ\left\{b_{2}^{3}\right\}$. Hence, by clause $(i)$ of Condition 3 , $\left\{b_{3}^{3}\right\} \succ\left\{b_{1}^{1}, b_{2}^{1}, b_{3}^{1}, b_{4}^{2}\right\}$. Continuing this process, we obtain $\left\{b_{m-2}^{3}\right\} \succ\left\{b_{1}^{1}, b_{2}^{1}, \ldots, b_{m-2}^{1}, b_{m-1}^{2}\right\}$. Repeatedly applying Lemma 7, we find $c_{i}^{1}$ such that $\left\{c_{i}^{1}\right\} \succ\left\{b_{i}^{1}\right\}$, for $i=1 \ldots m-2$ such that $\left\{b_{m-2}^{3}\right\} \succ\left\{c_{1}^{1}, c_{2}^{1}, \ldots, c_{m-2}^{1}, b_{m-1}^{2}\right\}$. This, combined with $\left\{b_{m-1}^{3}\right\} \sim\left\{b_{m-1}^{2}, b_{m-2}^{3}\right\},\left\{b_{m-1}^{2}\right\} \sim$ $\left\{b_{1}^{0}, b_{m-2}^{1}\right\},\left\{b_{m-1}^{2}\right\} \succ\left\{b_{m-2}^{3}\right\}$ and clause $(i)$ of Condition 3, implies that:

$$
\left\{b_{m-1}^{3}\right\} \succ\left\{c_{1}^{1}, c_{2}^{1}, \ldots, c_{m-2}^{1}, b_{m-2}^{1}, b_{m-1}^{0}\right\} .
$$

By Averaging and Restricted Independence:

$$
\left\{b_{m-1}^{3}\right\} \succ\left\{c_{1}^{1}, \ldots, c_{m-3}^{1}, b_{m-3}^{1}, b_{m-2}^{0}, b_{m-1}^{0}\right\} .
$$

By repeatedly combining Averaging and Restricted Independence in this way, one is led to the conclusion that:

$$
\left\{b_{m-1}^{3}\right\} \succ\left\{c_{1}^{1}, b_{1}^{1}, b_{2}^{0}, b_{3}^{0}, \ldots, b_{m-1}^{0}\right\} .
$$

Repeatedly applying Lemma 7, one finds $d_{i}^{1}$ such that $\left\{d_{i}^{1}\right\} \prec\left\{b_{i}^{0}\right\}$, for $i=2 \ldots m-1$ for which:

$$
\left\{b_{m-1}^{3}\right\} \succ\left\{c_{1}^{1}, b_{1}^{1}, d_{2}^{0}, d_{3}^{0}, \ldots, d_{m-1}^{0}\right\} .
$$

Repeatedly applying Averaging and Restricted Independence, we obtain:

$$
\left\{b_{m-1}^{3}\right\} \succ\left\{a_{1}, a_{2}, \ldots, a_{m-2}, \ldots, a_{m-1}, a_{m}\right\}=A .
$$


Step 9. Trivially, $\left\{b_{m-1}^{4}\right\}=\left\{b_{m-1}^{3}\right\} \succ A$.

Steps 6 to 9 can clearly be repeated infinitely often using the same argument. This remark completes the proof of (iii). Now, using Certainty Equivalence, let $x$ be a consequence such that $A \sim\{x\}$. Since the function $u$ found in Theorem 2 represents $\succsim$ as per (1), one has $u\left(b_{1}^{i}\right) \leq u(x) \leq u\left(b_{m-1}^{i}\right)$ for every $i$. Now it is easy to check that the sequences $\left\{u\left(b_{h}^{i}\right)\right\}$ for every $h$ are just like the sequences in Lemma 4 . Because of this Lemma, one has:

$$
\lim _{t \rightarrow \infty} u\left(b_{1}^{t}\right)=\lim _{t \rightarrow \infty} u\left(b_{m-1}^{t}\right)=\sum_{a \in A} \frac{u(a)}{\# A} .
$$

Hence, $u(x)=\sum_{a \in A} \frac{u(a)}{\# A}$. By transitivity, $A \succsim\{g\} \Longleftrightarrow\{x\} \succsim\{g\} \Longleftrightarrow \sum_{a \in A} \frac{u(a)}{\# A} \geq u(g)$.

\subsection{Proof of Theorem 1.}

Proof. From Theorem 4, we know that if $\succsim$ is an ordering on $\mathcal{P}(X)$ satisfying Averaging, Restricted Independence, Richness and the Archimedean axiom, then there exists a function $u: X^{\prime} \rightarrow \mathbb{R}$ such that, for all sets $A$ and $B \in \mathcal{P}\left(X^{\prime}\right)$, one has $A \succsim B \Longleftrightarrow \sum_{a \in A} \frac{u(a)}{\# A} \geq \sum_{b \in B} \frac{u(b)}{\# B}$. If $M(X)=m(X)=\varnothing$ so that $X=X^{\prime}$, then the proof is done. Assume first that $M(X) \neq \varnothing$ and let $t$ be a consequence in $M(X)$. We have $\{t\} \succ\{x\}$ for every $x \in X^{\prime}$. We first show that the image of $X^{\prime}$ under $u$, denoted $u\left(X^{\prime}\right)$, is a set of real numbers that is bounded from above. That is, there is a real number $\bar{b}$ such that $u(x) \leq \bar{b}$ for all $x \in X^{\prime}$. Suppose by way of contradiction that $u\left(X^{\prime}\right)$ is not bounded and consider, thanks to Proposition 7 , consequences $a, c_{0}$ and $b \in X^{\prime}$ such that $\{a\} \prec\left\{c_{0}\right\} \prec\{b\}$. By Averaging and transitivity, one has $\{a\} \prec\left\{a, c_{0}\right\} \prec\left\{c_{0}\right\} \prec\left\{c_{0}, b\right\}$ and, by Theorem 4 , one has $u(a)<\frac{u\left(c_{0}\right)+u(b)}{2} \Longleftrightarrow u(a)+u(a)<u\left(c_{0}\right)+u(b)$. Since $u\left(X^{\prime}\right)$ is unbounded, there is a real number $u^{\prime} \in u\left(X^{\prime}\right)$ such that $u^{\prime}+u(a) \geq u\left(c_{0}\right)+u(b)$. Since $u^{\prime} \in X^{\prime}$, there is a $c \in X^{\prime}$ such that $u(c)=u^{\prime}$. By Theorem 4 , one has $\{c, a\} \succsim\left\{c_{0}, b\right\} \succ\left\{c_{0}, a\right\}$. If follows from Richness that is some $c_{1}$ such that $\left\{c_{1}, a\right\} \sim\left\{c_{0}, b\right\}$. Since $\{a\} \prec\{b\}$, it follows from Restricted Independence that $\left\{c_{1}\right\} \succ\left\{c_{0}\right\}$. This procedure, initiated by finding $c_{0}$ and $c_{1}$, can clearly be iterated at infinitum. It therefore generates a sequence $c_{k}$, for $k=0, \ldots$, of consequences in $X^{\prime}$ such that $\left\{c_{k}, a\right\} \sim\left\{c_{k-1}, b\right\}$ for $k=1, \ldots$, . By assumption, $\{t\} \succ$ $\left\{c_{k}\right\}$ for every $k$ so that the sequence is bounded by $t$. Hence the fact that the sequence $\left\{c_{k}\right\}$ for $k=0,1 \ldots$ is infinite contradicts the Archimedean axiom. Analogously, starting from the assumption that $m(X) \neq \emptyset$, one can deduce that $u\left(X^{\prime}\right)$ is bounded from below. Since the set of real numbers $u\left(X^{\prime}\right)$ is either bounded from above and/or from below, it has a least upper bound and/or a greatest lower bound. We therefore extend $u$ to $X$ by defining, for every $t \in M(X)$ (if any), $u(t)=\sup _{x \in X^{\prime}} u(x)$ and, for every $s \in m(X)$ (if any), $u(s)=\inf _{x \in X^{\prime}} u(x)$. We now show that $u$ extended in this fashion represents $\succsim$ as per (1) on the whole set $X$ (and not only on $X^{\prime}$ ). By definition $u(t)>u(x)>u(s)$ for all $t \in M(X), x \in X^{\prime}$ and $s \in m(X)$, and $u$ represents $\succsim$ as per (1) on $X^{\prime}$ by Theorem 4 . Take any $x \in X^{\prime}$. By Certainty Equivalence, there are consequences $b$ and $c \in X$ such that $\{b\} \sim\{x, t\}$ and $\{c\} \sim\{x, s\}$. By Averaging and transitivity, we have $\{s\} \prec\{c\} \sim\{x, s\} \prec\{x\} \prec\{x, t\} \sim\{b\} \prec\{t\}$ so that both $b$ and $c$ belong to $X^{\prime}$. We therefore only need to show that $\frac{u(x)+u(t)}{2}=u(b)$ and $\frac{u(x)+u(s)}{2}=u(c)$. The argument being symmetric, we only prove that $\frac{u(x)+u(t)}{2}=u(b)$. By contradiction, suppose first that $\frac{u(x)+u(t)}{2}<u(b)$. By Certainty Equivalence, there exists some $b_{1} \in X$ such that $\left\{b_{1}\right\} \sim\{x, b\}$. By Averaging 
$\{x\} \prec\left\{b_{1}\right\} \prec\{b\}$ and, therefore, $b_{1} \in X^{\prime}$. By Theorem 4 , the statement $\left\{b_{1}\right\} \sim\{x, b\}$ can be written as $u\left(b_{1}\right)=\frac{u(x)+u(b)}{2}$. Define recursively $b_{n}$ by $\left\{b_{n}\right\} \sim\left\{b_{n-1}, b\right\}$ for $n=2$,.... Since $\{t\} \succ\{b\} \succ\left\{b_{n}\right\} \succ\left\{b_{n-1}\right\}$ by Averaging and transitivity, we have that $b$ and $b_{n} \in X^{\prime}$ so that, by Theorem $4, u\left(b_{n}\right)=\frac{u\left(b_{n-1}\right)+u(b)}{2}=\frac{\frac{1}{2}\left[u\left(b_{n-2}\right)+u(b)\right]+u(b)}{2}=\ldots=\frac{u(x)}{2^{n-1}}+\frac{2^{n-1}-1}{2^{n-1}} u(b)$. Hence, for $n$ large enough, $\left.u\left(b_{n}\right) \in\right] \frac{u(x)+u(t)}{2}, u(b)\left[\right.$. Now, we know that $\{b\} \sim\{x, t\} \succ\left\{b_{n}\right\} \succ\left\{b_{n-1}, x\right\}$. By Richness, there exists $t^{\prime}$ such that $\left\{x, t^{\prime}\right\} \sim\left\{b_{n}\right\}$. Since $\{x, t\} \succ\left\{b_{n}\right\} \sim\left\{x, t^{\prime}\right\}$, it follows from Restricted Independence that $\left\{t^{\prime}\right\} \prec\{t\}$. Hence $x, b_{n}$ and $t^{\prime} \in X^{\prime}$ so that, by Theorem 4 , $\frac{u\left(t^{\prime}\right)+u(x)}{2}=u\left(b_{n}\right)>\frac{u(t)+u(x)}{2}$. Yet this inequality is incompatible with the definition of $u(t)$ as $u(t)=\sup _{x \in X^{\prime}} u(x)$.

\subsection{Proof of Theorem 3.}

Proof. We know from Proposition 2 that a UEU criterion satisfies Averaging and Restricted Independence on any environment. Conversely, let $X$ be a connected separable topological space and let $\succsim$ be an ordering of $\mathcal{P}(X)$ satisfying the Continuity axiom as well as Averaging and Restricted Independence. We will prove that, under these conditions, $\succsim$ satisfies Richness and the Archimedean axiom. Using Theorems 2, 4 and 1, the conclusion that $\succsim$ is a UEU criterion will then follow immediately. We first notice that, under Averaging, if the sets $B(A)=\{x \in$ $X:\{x\} \succsim A\}$ and $W(A)=\{x \in X: A \succsim\{x\}\}$ are closed in $X$ for every $A$, then so are the sets $\widetilde{B}(A)=\{x \in X: A \cup\{x\} \succsim A\}$ and $\widetilde{W}(A)=\{x \in X: A \succsim A \cup\{x\}\}$. To see that, assume by contraposition that, say, $\widetilde{B}(A)$ is not closed (the argument for $\widetilde{W}(A)$ is similar). Then, there exists a sequence $\left\{x^{t}\right\}, t=1, \ldots$ converging to some limit $x$ such that:

$$
A \cup\left\{x^{t}\right\} \succsim A
$$

for all $t$ and

$$
A \succ A \cup\{x\}
$$

where the last strict ranking is obtained from the assumption that $\succsim$ is complete. Since $\succsim$ is also reflexive, this strict ranking implies therefore that $x \notin A$. By Averaging one has therefore:

$$
A \succ\{x\}
$$

Now, since $A$ is finite, and $\left\{x^{t}\right\}$ is a sequence converging to $x$, either $\left\{x^{t}\right\}$ is a finite sequence or $\left\{x^{t}\right\}$ is infinite. If $\left\{x^{t}\right\}$ is finite, then, by definition, there exists some $s \leq t$ for which $x^{s}=x \notin X$. But given Averaging, this is incompatible with the definition of the sequence $\left\{x^{t}\right\}$ as satisfying $A \cup\left\{x^{t}\right\} \succsim A$ for every $t$. Hence we must conclude that $x^{t}$ is infinite. If this is the case, there must exists, since $A$ is finite, an infinite subsequence $\left\{\widetilde{x}^{t}\right\}$ of $\left\{x^{t}\right\}$ converging to $x$ and such that $\widetilde{x}^{t} \notin A$ for every $t$. Since for every $t$, we have:

$$
A \cup\left\{\widetilde{x}^{t}\right\} \succsim A
$$

it follows from Averaging that we also have:

$$
\left\{\widetilde{x}^{t}\right\} \succsim A
$$

Given (10), this gives us the required contradiction of the closedness of the set $B(A)$. We now prove that $\succsim$ satisfies Richness and the Archimedean axiom.

Richness: Consider any set $B \in \mathcal{P}(X)$ and, without loss of generality, write it as $B=$ $\left\{b_{1}, \ldots, b_{\# B}\right\}$ with $\left\{b_{h}\right\} \precsim\left\{b_{h+1}\right\}$ for $h=1, \ldots, \# B-1$. By Averaging (and specifically the Gardenförs principle) one has that $B \succsim\left\{b_{1}\right\}$ and $\left\{b_{\# B}\right\} \succsim B$ so that none of the (closed 
under Continuity) sets $\{x \in X:\{x\} \succsim B\}$ and $\{x \in X: B \succsim\{x\}\}$ is empty. Since $\succsim$ is complete, $X=\{x \in X:\{x\} \succsim B\} \cup\{x \in X: B \succeq\{x\}\}$. Since $X$ is connected and can therefore not be written as the union of two disjoint non-empty closed sets, there exists $x \in\{x \in X:\{x\} \succsim B\} \cap\{x \in X: B \succeq\{x\}\}$. By definition such a $x$ satisfies $\{x\} \sim B$. Hence $\succsim$ satisfies Certainty Equivalence. Consider now any sets $A$ and $B$ in $\mathcal{P}(X)$ and consequences $c^{*}$ and $c_{*} \in X$ such that $A \cup\left\{c^{*}\right\} \succsim B \succsim A \cup\left\{c_{*}\right\}$ holds. Since, as was just shown, $\succsim$ satisfies Certainty Equivalence, there are consequences $b$ and $b(c) \in X$ (for all $c \in X$ ) such that $\{b(c)\} \sim A \cup\{c\}$ and $B \sim\{b\}$. By Continuity, the restriction of the ordering $\succsim$ to singletons is continuous. Hence, by Debreu [32] Theorem 1, there exists a continuous function $f: X \longrightarrow \mathbb{R}$ such that $f(x) \geq f(y)$ if and only if $\{x\} \succsim\{y\}$ for every $x, y \in X$. Define therefore the function $h: X \rightarrow \mathbb{R}$ by $h(c)=f(b(c))-f(b)$. We first notice that the function $h$ so defined is continuous if $f$ is. Indeed, suppose $h$ is not continuous. This means that there exists a sequence $\left\{c_{t}\right\}$ of elements of $X$ converging to some element $\bar{c}$ of $X$ for which the sequence $\left\{h\left(c_{t}\right)\right\}$ of real numbers does not converge to the number $h(\bar{c})$. The fact that sequence $\left\{h\left(c_{t}\right)\right\}$ does not converge to $h(\bar{c})$ means that there is some strictly positive number $\varepsilon$ for which it is the case that, for every $t^{\prime}$, there is some $t^{\prime \prime}>t^{\prime}$ for which one has:

$$
\begin{aligned}
\left|h\left(c_{t^{\prime \prime}}\right)-h(\bar{c})\right| & >\varepsilon \\
\left|f\left(b\left(c_{t^{\prime \prime}}\right)\right)-f(b(\bar{c}))\right| & \Longleftrightarrow \varepsilon
\end{aligned}
$$

Consider now the open (in $\mathbb{R}$ ) set $A=\{x \in \mathbb{R}:|x-f(b(\bar{c}))|<\varepsilon\}$. This set obviously contains the number $f(b(\bar{c}))$. Because $f$ is a continuous function from $X$ (a topological space) to $\mathbb{R}$, the inverse image $f^{-1}(A)=\{c \in X:|f(c)-f(b(\bar{c}))|<\varepsilon\}$ is open and obviously contains $\bar{c}$. Because the sequence $\left\{c_{t}\right\}$ converges to $\bar{c}$, there exists a $t^{\prime}$ such that $c_{t^{\prime \prime}} \in f^{-1}(A)$ for all $t^{\prime \prime}>t^{\prime}$. That is, there exists a $t^{\prime}$ such that $\left|f\left(b\left(c_{t^{\prime \prime}}\right)\right)-f(b(\bar{c}))\right|<\varepsilon$ for all $t^{\prime \prime}>t^{\prime}$, contradicting (11). Hence $h$ is indeed a continuous function. By assumption, we have $h\left(c^{*}\right) \geq 0$ and $h\left(c_{*}\right) \leq 0$. Since $X$ is connected, we can appeal to the version of the Intermediate Value Theorem provided in [33] (Proposition 8-11, p. 182) to exhibit a consequence $\bar{c}$ such that $h(\bar{c})=f(b(\bar{c}))-f(b)=0$. By definition, $\bar{c}$ is such that $A \cup\{\bar{c}\} \sim\{b\} \sim B$, as required.

Archimedean axiom: If it is impossible to construct one of the standard sequences as in the antecedent clause of the Archimedean axiom, then the proof is (trivially) over. Assume therefore that such a sequence exists (we only provide the argument for the ascending sequence) and, therefore, that $a$ and $b$ are two consequences in $X$ satisfying $\{a\} \succ\{b\}$ for which one has, for a sequence of consequences $\left\{c_{t}\right\}_{t \in \mathbb{N}_{+}}$:

$$
\left\{c_{t}, a\right\} \sim\left\{c_{t+1}, b\right\} .
$$

We notice that, by Restricted Independence, one has $\left\{c_{t+1}\right\} \succ\left\{c_{t}\right\}$ for every $t \in \mathbb{N}_{++}$. We will show that if such a sequence is infinite, then it is unbounded from above. For this task, let $C_{t}=\left\{x \in X:\{x\} \prec\left\{c_{t}\right\}\right\}$. By Continuity, $C_{t}$ is open because it is the complement in $X$ (by completeness) of a closed set. Hence the set $C=\bigcup_{t \in \mathbb{N}_{++}} C_{t}$ is open and if $z \in X \backslash C$, then $\{z\} \succsim\left\{c_{t}\right\}$ for every $t$. We will show that the set $X \backslash C$ of upper bounds for the sequence $\left\{c_{t}\right\}_{t \in \mathbb{N}_{+}}$is empty. By contradiction, let $z \in X / C$. By Restricted Independence, one has (using (12):

$$
\{z, a\} \succ\{z, b\} \succsim\left\{c_{t+1}, b\right\} \sim\left\{c_{t}, a\right\}
$$

for every $t$. Since, as was proved above, Richness holds, there exists some $w \in X$ such that $\{w, a\} \sim\{z, b\}$. By Restricted Independence, $\{z\} \succ\{w\}$ and $w \in X / C$ because $\{w\} \succsim\left\{c_{t}\right\}$ for all $t$. Now the set $\{v:\{v\} \succ\{w\}\}$ is open by Continuity, contains $z$ and is a subset of $X / C$ by transitivity. This means that that $X / C$ is open because it is a union of open sets. But this contradicts the assumption that $X$ is connected and, as a result, can not be written as a union of two disjoint open sets. 


\subsection{Proof of Proposition 5.}

Proof. It is straightforward to verify that if $\succsim^{1}$ and $\succsim^{2}$ are two orderings on $\mathcal{P}(X)$ that can be represented as per (1) for some real-valued continuous functions $u^{1}$ and $u^{2}$ (having both $X$ as domain) such that $u^{1}(x)=\Psi\left(u^{2}(x)\right)$ holds for every $x \in X$ for some continuous, increasing and concave real valued function $\Psi$ having the image of $\mathbb{R}$ under $u^{2}$ as domain, then $\succsim^{1}$ is at least as ambiguity/uncertainty averse as $\succsim^{2}$ as per Definition 1. Conversely, let $\succsim^{1}$ and $\succsim^{2}$ be two orderings on $\mathcal{P}(X)$ that can be represented as per (1) for the real-valued functions $u^{1}$ and $u^{2}$ having both $X$ as domain and let $\succsim^{1}$ be at least as ambiguity/uncertainty averse as $\succsim^{2}$ as per Definition 1. We first notice if $\succsim^{1}$ is at least as ambiguity/uncertainty averse as $\succsim^{2}$ as per Definition 1, then $\succsim^{1}$ and $\succsim^{2}$ must provide the same ranking of singletons. Indeed, suppose by contradiction that there are consequences $x$ and $y \in X$ for which $\{x\} \succsim^{1}\{y\}$ holds but $\{x\} \succsim^{2}\{y\}$ does not hold. Since $\succsim^{2}$ is complete, one must have $\{y\} \succ^{2}\{x\}$ and, since $\succsim^{1}$ is at least as ambiguity/uncertainty averse as $\succsim^{2}$ as per Definition 1, one must have $\{y\} \succ^{1}\{x\}$, a contradiction. Now, the functions $u^{1}$ and $u^{2}$ are both numerical representations of the orderings of $X$ induced by the restriction of $\succsim^{1}$ and $\succsim^{2}$ to singletons. Since these orderings are the same, there must be some increasing real valued function $\Psi$ having the image of $\mathbb{R}$ under $u^{2}$ as domain for which $u^{1}(x)=\Psi\left(u^{2}(x)\right)$ holds for every $x \in X$. Moreover, $\Psi$ must be continuous if $u^{1}$ and $u^{2}$ are continuous. We only need to show that $\Psi$ must be concave as well. By contradiction, suppose it is not concave so that, for some $a$ and $b$ in the domain of $\Psi$ and some $\lambda \in] 0,1[$ for which $\lambda a+(1-\lambda) b$ is in the domain of $\Psi$, one has $\Psi(\lambda a+(1-\lambda) b)<\lambda \Psi(a)+(1-\lambda) \Psi(b)$. Because $\Psi$ is continuous, there is no loss of generality in assuming $\lambda=1 / 2$. Hence, one has

$$
\Psi\left(\frac{a+b}{2}\right)<\frac{\Psi(a)+\Psi(b)}{2} .
$$

Because $a, b$ and $\frac{a+b}{2}$ belong to the domain of $\Psi$ taken to be the image of $X$ under $u^{2}$, there are consequences $x, y$ and $z \in X$ such that:

$$
u^{2}(x)=a, u^{2}(y)=\frac{a+b}{2} \text { and } u^{2}(z)=b .
$$

Because the function $u^{1}$ defined by $u^{1}(c)=\Psi\left(u^{2}(c)\right)$ for every $c \in X$ represents the ordering $\succsim^{1}$ as per (1), inequality (13) implies that:

$$
\{y\} \prec^{1}\{x, z\}
$$

On the other hand, the fact that the function $u^{2}$ represents $\succsim^{2}$ as per (1) together with (14) imply that:

$$
y \sim^{2}\{x, z\}
$$

in contradiction with the fact that $\succsim^{1}$ is at least as uncertainty/ambiguity averse as $\succsim^{2}$.

\section{Acknowledgements}

We are indebted, with the usual disclaiming qualification, to Peter Wakker for his helpful and encouraging comments and to David Krantz for very useful discussions about example 3 in Section 6. The second author would also like to acknowledge the hospitality of the Indian Statistical Institute in New Delhi where some of the work leading to this paper was conducted.

\section{References}

[1] J. V. Neumann, O. Morgenstern, Theory of Games and Economic Behavior, Princeton University Press, Princeton, NJ, USA, 1947. 
[2] J. L. Savage, The Foundation of Statistics, Wiley, New York, 1954.

[3] S. Barberà, W. Bossert, P. K. Pattanaik, Ranking sets of objects, in: S. Barberà, P. Hammond, C. Seidl (Eds.), Handbook of Utility Theory, vol. 2: Extensions, Kluwer, Dordrecht, 2004, pp. 893-977.

[4] D. S. Ahn, Ambiguity without a state space, Review of Economic Studies 75 (2008) 3-28.

[5] W. Olszewski, Preferences over sets of lotteries, Review of Economic Studies 74 (2007) $567-595$.

[6] P. K. Pattanaik, B. Peleg, An axiomatic characterization of the lexicographic maximin extension of an ordering over a set to the power set, Social Choice and Welfare 1 (1984) $113-122$.

[7] N. Baigent, Y. Xu, Decisions under complete ignorance, mimeo,Institute of public economics, Graz university (2004).

[8] S. Nitzan, P. K. Pattanaik, Median-based extensions of an ordering over a set to the power set: An axiomatic characterization, Journal of Economic Theory 34 (1984) 252-261.

[9] J. M. Keynes, A treatise on probabilities, Macmillan, New York, 1921.

[10] E. T. Jaynes, Probability Theory: the Logic of Science, Cambridge University Press, Cambridge UK, 2003.

[11] P. C. Fishburn, Even-chance lotteries in social choice theory, Theory and Decision 3 (1972) $18-40$.

[12] D. Krantz, R. D. Luce, P. Suppes, A. Tversky, Foundations of Measurement, vol. 1, Academic Press, New York London, 1971.

[13] E. D. Bolker, Functions resembling quotient of measures, Transaction of the American Mathematical Society 124 (1966) 292-312.

[14] E. D. Bolker, A simultaneous axiomatization of utility and subjective probabilities, Philosophy of Sciences 34 (1967) 333-340.

[15] R. Jeffrey, The Logic of Decision, University of Chicago Press, Chicago, 1965, reprint 1983.

[16] J. Broome, Bolker-jeffrey expected utility theory and axiomatic utilitarianism, Review of Economic Studies 57 (1990) 477-502.

[17] S. Barberà, B. Dutta, A. Sen, Strategy-proof social choice correspondences, Journal of Economic Theory 101 (2001) 374-394.

[18] J. P. Benoît, Strategic manipulation in voting games when lotteries and ties are permitted, Journal of Economic Theory 102 (2002) 421-436.

[19] S. Ozyürt, R. Sanver, A general impossibility result on strategy-proof social choice hyperfunctions, Games and Economic Behavior 66 (2009) 880-892.

[20] B. Peleg, H. Peters, Nash consistent effectivity functions through lottery models, Games and Economic Behavior 65 (2009) 503-515.

[21] D. Scott, Measurement structure and linear inequalities, Journal of Mathematical Psychology 1 (1964) 233-247. 
[22] E. W. Adams, Elements of a theory of inexact measurement, Philosophy of Science 32 (1965) 205-228.

[23] P. Fishburn, Utility Theory for Decision Making, John Wiley, New York, 1970.

[24] F. Gul, W. Pesendorfer, Temptation and self-control, Econometrica 69 (2001) 1403-1435.

[25] P. A. Gärdenfors, On definitions of manipulation of social choice function, in: J. Laffont (Ed.), Aggregation and Revelation of Preferences, North Holland, Amsterdam, 1976.

[26] K. Nehring, C. Puppe, Continous extension of an order on a set to the power set, Journal of Economic Theory 68 (1996) 456-479.

[27] C. Puppe, Freedom of choice and rational decisions, Social Choice and Welfare 12 (1995) $137-154$.

[28] G. Debreu, Topological methods in cardinal utility theory, in: S. K. K. J. Arrow, P. Suppes (Eds.), Mathematical methods in the social sciences, 1959; proceedings, Stanford University Press, Stanford, 1960, pp. 16-26.

[29] E. W. Adams, R. F. Fagot, A model of riskless choice, Behavioral Science 4 (1959) 1-10.

[30] P. Wakker, The algebraic versus the topological approach to additive representations, Journal of Mathematical Psychology 32 (1988) 421-435.

[31] A. Mas-Colell, M. D. Whinston, J. R. Green, Microeconomic Theory, Oxford University Press, Oxford, UK, 1995.

[32] G. Debreu, Representation of a preference ordering by a numerical function, in: R. L. D. R. M. Thrall, C. H. Coombs (Ed.), Decision Processes, Wiley, New York, 1954, pp. 159-165.

[33] H. L. Royden, Real Analysis, 3rd Edition, Macmillan, New York, 1988. 